\title{
A novel plasma membrane-phillic graphene oxide nanocarrier for neuropeptide delivery to generate tolerogenic dendritic cells in GVHD Immunotherapy
}

Xiaohui Wang ( $D$ lovechina1980@163.com)

Institute of Health Service and Transfusion Medicine, Beijing https://orcid.org/0000-0003-3091-0219

\section{Sujing Sun}

Institute of Health Service and Transfusion Medicine

Chulin He

Zhuhai People's hospital, Guangdong

Chenyan Li

BGI college, Zhengzhou University

Wenyuan Bai

BGI college, Zhengzhou University

\section{Yulong Zhang}

Institute of Health Service and Transfusion Medicine

Minwei He

Institute of Health Service and Transfusion Medicine

Liping Lv

Institute of Health Service and Transfusion Medicine

Yanrong Dong

Institute of Health Service and Transfusion Medicine

\section{Ping Ma}

Institute of Health Service and Transfusion Medicine

Qianqian Zhou

Institute of Health Service and Transfusion Medicine

Linsheng Zhan

Institute of Health Service and Transfusion Medicine

\section{Article}

Keywords: Graphene Oxide, Tolerogenic Dendritic Cells, Urocortin, Acute GVHD

Posted Date: December 9th, 2021

DOl: https://doi.org/10.21203/rs.3.rs-1148600/v1 
License: (c) (i) This work is licensed under a Creative Commons Attribution 4.0 International License. Read Full License 


\section{Abstract}

The prevention and the treatment of graft-versus-host disease (GVHD) remains a barrier to allogeneic hematopoietic stem cell transplantation (allo-HSCT). Tolerogenic dendritic cells (TDCs) have become a hotspot in GVHD prevention despite the poor induction efficiency. Herein, we designed a novel sizedependent platform of graphene oxide (GO) nanosheets for neuropeptide delivery for the purpose of TDC generation. $\mathrm{GO}$ with a lateral size $\mathbb{1} 1 \mu \mathrm{m}$ (L-GO) showed strong affinities to DC membrane, which effectively promoted the recognition between neuropeptide, urocortin (UCN) and its receptor CRHR2 and in turn benefited TDC generation through PKA C/CREB phosphorylation. Simultaneously, L-GO also elevated the expression of CCR7 and enhanced the migration ability of TDCs by mediating cytoskeleton reorganization. In vivo experiments offered direct evidence that TDCs inducted by UCN@L-GO exhibited efficient migration to lymph nodes, abundant generation of Treg, a significant decrease of proinflammatory cytokines and in turn excellent efficiency in GVHD relief. In the current study, we proposed an innovative $\mathrm{GO}$ nanosheets based cytomembrane-targeted platform for neuropeptide delivery and subsequent TDC generation. In the meantime, the promoted mobility of TDC pulsed by GOs ensured the high homing efficiency to secondary lymph nodes, attributing to GVHD treatment in vivo. Thus, such work provided a promising strategy that might be applicable more broadly to delivery systems for receptor-mediated drugs, and could meet the changing demand of nanotechnology-based immunotherapy.

\section{Introduction}

Allogeneic hematopoietic stem cell transplantation (allo-HSCT) has become a first-line treatment for many types of malignancies, such as leukemia, lymphoma, and multiple myeloma ${ }^{1}$. Graft-versus-host disease (GVHD) is a serious and life-threatening complication that undergoes allo-HSCT with incidence rates ranging from 20 to $80 \%{ }^{2}$, which limits the efficiency of allo-HSCT. Generally, GVHD arises from the activation of donor $T$ lymphocytes after APCs presentation and attack the recipient's tissues, including the skin, liver, gastro intestinal tract and nervous system ${ }^{3,4}$. Based on the guidelines of American Society of Blood and Marrow Transplantation, corticosteroids are the mainstay treatments for GVHD. However, systemic corticosteroid showed limited overall complete response and a series of sequelae ${ }^{2}$, like high incidence of steroid-refractory ${ }^{2}$. So far, therapies targeting immune cells and immune cytokines have proved to be effective in GVHD treatment. In particular, adoptive immunotherapy of tolerogenic dendritic cells (TDCs), regulatory T cells (Tregs) and mesenchymal stem cells (MSCs) showed positive effect in the relief of GVHD by activating T cell deletion ${ }^{5,6}$. MSCs can interact with innate immune cells to downregulate immunity, but the lung-accumulated distribution of MSCs after transplantation limits its function. Tregs were also threatened by the targeted gene editing strategy ${ }^{2,7}$.

DCs are known as the strongest antigen-presenting cells (APCs) that accommodate immune response by eliciting primary $T$ cell response and modulating immune tolerance ${ }^{8-10}$. In the initial stage of GVHD, DCs presented host antigens which participated in donor T cell expansion, mediated GVHD and caused severe 
damage to the liver, skin and intestines ${ }^{4,11}$. DCs have excellent ability to lead T cells to exert immune cell response or induce immune tolerance. They can induce peripheral tolerance to relieve GVHD by deleting cytotoxic T cells and upgrading Treg expansion ${ }^{12-14}$. Preclinical data from murine BMT (bone marrow transplantation) model systems also showed that recipient DCs improved survival by decreasing the severity of GVHD. Therefore, induction of TDCs, especially recipient TDCs, may impair the immuneregulatory function and facilitate the generation of Tregs. Thus, TDC generation could be a potent candidate for GVHD treatment ${ }^{15}$.

Many protocols have been established to generate TDCs in vitro. Treatment of DCs with broad immunosuppressants, such as glucocorticoids, rapamycin and cyclosporine, could contribute to prevention in animal models of allograft rejection and autoimmune disorders. However, such agents may exert such adverse effects as increased infection, disordered metabolism, peptic ulcer and hypertension ${ }^{16}$. Cytokines, such as IL-10 and TGF- $\beta$, were also used in DCs pretreatment and showed suppressive efficacy in animal models. Unfortunately, these TDCs were not stable since they could produce proinflammatory cytokines upon introduction of a second stimuli ${ }^{17,18}$. However, even in such a semi-activation state, these TDCs showed slight elevation in mobility, which notably limited its performance.

Recently, neuropeptides found in neural tissues and produced by immune cells under inflammation have been rediscovered as potential candidates against autoimmune diseases and transplantation complications. Recent research demonstrated that neuropeptides, such as urocortin (UCN), vasoactive intestinal peptide (VIP), a-melanocyte-stimulating hormone(a-MSH), and adrenomedullin (AM), all had potent effects in inducing immune tolerance ${ }^{19}$. UCN, a member of the corticotropin-releasing hormone $(\mathrm{CRH})$ family, acted as a broad neuromodulator and was demonstrated to be a potential balance factor in immune tolerance induction ${ }^{20}$ through an indirect anti-inflammation effect mediated by the hypothalamus-pituitary-adrenal (HPA) axis ${ }^{21}$. AM is believed to act as an endogenous immunomodulatory factor with predominantly anti-inflammatory effects ${ }^{22}$. Experiments also showed that after treatment with VIP, DCs could induce a significant decrease in expressions of co-stimulatory molecules and in the production of anti-inflammatory cytokines. Notably, these TDCs kept their immature phenotype even when exposed to an inflammatory environment, which is of great importance for GVHD treatment ${ }^{23}$. Despite easy availability and broad regulation of autoimmunity, the application of neuropeptides as immunomodulators in vivo still is hindered by easy degradation and poor penetration.

In recent years, nanotechnology is fast-developing and provides a novel platform for biomedical applications. In particular, engineering nanomaterials for the delivery of cargos or regulation of the expression profile of DCs during transplantation have been regarded as a potential means of immunomodulation for tolerogenic outcomes. Graphene oxide (GO), a typical 2D nanomaterial that was reported in 2004 for the first time, has attracted much attention in biomedical research ${ }^{24}$. Despite the varying lateral dimensions, $\mathrm{GO}$ has a relatively large specific surface area and processes excellent capability for bioactive molecule interaction. Therefore, besides the efforts of applying GO as biosensor 
assay in detecting infectious disease 25,26 , it is always utilized as a nanoscale vehicle for targeted drug delivery in vivo thanks to its limited cytotoxicity and high biocompatibility ${ }^{27,28}$. It is well documented that large-sized GO (normally $1 \mu \mathrm{m}$ or above) prefers to adhere onto the cell plasma membrane, unlike its small counterpart that tends to be internalized by immunocytes through endocytosis. Moreover, it is able to modulate the $\mathrm{DC} / \mathrm{T}$ interactions ${ }^{29}$ and shows some degree of immunosuppression or T cell tolerance. On the other hand, it is found that the most of the receptors of neuropeptides belongs to the G-proteincoupled receptor (GPCR) family, which located on the cell membrane ${ }^{19}$. Given the potential ability of antiinflammation and immunoregulation, it is intriguing to explore the combination of $\mathrm{GO}$ and neuropeptides in TDC induction. Therefore, here we designed a GO-based therapeutic platform to carry neuropeptides to the surface of immature DCs for TDC generation, and investigate its behavior and possible mechanism in GVHD treatment. After the most effective neuropeptides were screened out from their candidates, in-depth exploration indicated that GO loading improved the migration ability of DCs, which facilitated the homing of TDCs to secondary lymph nodes. An acute GVHD model was employed to investigate the efficacy of as-prepared TDCs. The Fluc+ T cells were used in our model for in-situ imaging, which ensured real-time observation of proliferation of donor T cells and efficiency of GVHD treatment. Our data indicated that the neuropeptide-GO complex could be used as a potent therapeutic candidate for the prevention of GVHD. More importantly, the current work proposed a cytomembrane-philic biomacromolecule delivery system with a novelty design.

\section{Results}

\section{Characterization of $\mathrm{GO}$ and its affinity to cell membrane.}

GO of different scales were synthesized as vehicles for the delivery of neuropeptides to DCs.

Transmission electron microscopy (TEM) observations showed that GOs were characterized by irregular polygon of different lateral sizes (Figure 1A). The hydrodynamic radii of GOs were measured by dynamic light scattering (DLS) as $103.1 \mathrm{~nm}$ (marked as S-GO) and $1192.1 \mathrm{~nm}$ (marked as L-GO), respectively (Supplementary Figure 1). Atomic force microscopy (AFM) revealed the average thickness of 1-2 nm, which aligned with the structural characteristics of monolayer 2D materials (Figure 1B). In order to determine the maximum allowable concentration of GOs in delivery, flow cytometry was performed to analyze the survival rates of imDCs after incubation with GOs for $48 \mathrm{~h}$. When the concentration of GOs was set as $15.6 \mu \mathrm{g} / \mathrm{ml}, \mathrm{GO}$ showed little toxicity with the survival rate of imDCs higher than $90 \%$ compared to the control group (Supplementary Figure 2).

GO was proved to have strong affinities to cell membrane and mediate contact through membrane interactions. According to these characteristics, GO was chosen as a good nanoscaffold for variety biomedical applications. Confocal Raman results showed that after co-culture with S-GO or L-GO for 48h, the accumulation of GO was observed in cell membrane, suggesting that both S-GO and L-GO had nonspecific affinities to accumulate in cell-rich areas including RAW264.7, Hela and DC. L-GO were mostly adhered to the membrane while S-GO showed a preference of locating in cytoplasm (Figure 1C). Cytomembrane is rich in transmembrane receptors, which is a critical component in cell properties and 
subtype switching. However, whether GO' affinities have any influence on membrane receptors or on the combination of ligands and receptors are still unknown. Here we chose RBCs as a lipid bilayer model because it has no organelles or nucleus, thus simplifying the interaction model. The surface of RBCs was first biotinylated to varying degrees to imitate receptors of different abundances on cytomembrane, and different concentrations of S-GO or L-GO were added to PE labeled avidin to allow physical adsorption. The binding process of avidin-biotin was used to simulate the combination that was dependent on the ligand and receptor (Figure 1D). The combining efficacy was reflected by the mean fluorescence intensity (MFI) of PE monitored by FACS. It is worth noting that even in the non-biotinylated group, the introduction of GO caused nonspecific affinity to RBCs (Supplementary Figure 3). Figure 1E gave direct evidence that GO-pretreatment could increase the binding affinity between PE-labeled avidin and biotinylated RBCs. Despite the presence of nonspecific binding, such improvement was much more significant in the case of L-GO than in its counterpart, especially in the low abundant biotin group ( 1 or $2 \mu \mathrm{g}$ biotin added in the labelling system). The specific binding of biotin-avidin was measured after deduction of the nonspecific binding. When $8 \mu \mathrm{g} \mathrm{GO}$ was added to the pretreated avidin, FACS results showed that S-GO and L-GO both increased MFI at each concentration of biotin, especially at a lower concentration (especially $1 \mu \mathrm{g}$ and 2 $\mu \mathrm{g}$ ), and that L-GO showed stronger improvement in MFI than S-GO did (Figure 1E). The results after nonspecific binding deduced are depicted as Figure $1 \mathrm{~F}$, in which case the GOs just acted as a promoter in the course of specific recognition of avidin and biotin. Take biotinylated RBCs ( $1 \mu \mathrm{g}$ group) as an example. the addition of different concentrations $(2,4$ and $8 \mu \mathrm{g})$ of S-GO showed 1.98-, 2.03- and 3.87-fold enhancements compared to the blank, respectively. Meanwhile, the corresponding results for the L-GO group were 2.58-, 3.87- and 4.71-fold, respectively. These results indicated that GOs' high affinity to cytomembrane facilitated the specific combination between the ligand and receptor, and that the largesized ones showed stronger enhancement (Figure 1F). As the current work was intended to explore TDCsbased immunotherapy in GVHD treatment, we then proceeded to the investigation of the interaction manner between GOs and DCs. Laser confocal was performed to validate the location of GOs on DC plasma membrane after $24 \mathrm{~h}$ of co-culture. S-GO and L-GO were labeled with FITC, and DCs were stained with rhodamine. Figure $1 \mathrm{G}$ shows that S-GO was mainly found in the cytoplasm of DCs, while L-GO was located at the edge of cells that were likely to correspond to the GOs associated with the plasma membrane, which was consistent with the phenomenon observed in the above RBCs-based model and literature. (Figure 1G).

\section{The screening of neuropeptides aiming at inducing TDCs}

Neuropeptides, mainly secreted by immune cells such as macrophages and monocytes, were considered capable of inducing immune tolerance with a broad anti-inflammatory effect. Neuropeptides UCN, a-MSH, VIP and AM were chosen as alternatives to induce the generation of TDCs firstly in a non-inflammatory microenvironment. Proinflammatory cytokines, such as IL-12 $\mathrm{p}^{70}, \mathrm{IL}-6, \mathrm{IL}-1 \beta$ and TNF- $a$ were measured by ELISA. It was found that neuropeptide incubation had little influence on cytokine secretion of DCs as shown in Supplementary Figure 4. Given the systemic inflammation in case of GVHD, we next investigated the performance of the four candidates in an inflammatory environment established by low- 
dose LPS co-incubation for $48 \mathrm{~h}$. In the case of $\mathrm{DC}_{\text {LPS }}$ group, stimulatory DCs (SDCs) were obtained with elevated proinflammatory cytokines secretion (Figure 2A). Meanwhile, in the presence of neuropeptide, the proinflammatory cytokines were found to be significantly decreased. Indeed, IL-12p ${ }^{70}$ secreted by DCs co-cultured with UCN declined by $61.8 \%$ percent compared with the LPS stimulated ones $(85.2 \pm 10.8 \mathrm{pg} / \mathrm{ml})$. IL-6 showed $80.3 \%$ decrease, while IL- $1 \beta$ was $64.8 \%$ and TNF-a was $82.4 \%$, indicating that UCN exhibited more effective suppression on proinflammatory cytokines secretion than other candidates (Figure 2A).

After UCN was selected as the most appropriate neuropeptides in the study, we stared to find out whether UCN was capable of inducing TDCs. Balb/c derived CD8 ${ }^{+} \mathrm{T}$ cells and C57BL/6J derived DCs were isolated separately. CD8+ T cells were stained with carboxyfluorescein diacetate succinimidyl ester (CFSE) and co-cultured with DCs at ratios of 2:1, 4:1 and 8:1 for 48h. Results showed that DCs with UCN treatment exhibited significant inhibition on cytotoxic $T$ cell proliferation. And the strongest suppression was observed at the ratio of $4: 1$ with a decrease of $52.6 \%$ compared to $\mathrm{DC}_{\mathrm{LPS}}$. Cytotoxic T-cell proliferation test demonstrated that TDC $\mathrm{UCN}_{\mathrm{CN}}$ decreased CD8+ T cell proliferation and induced T cell anergy (Figure 2B, 2C). CD40/80/86 were employed to characterize the maturity of DCs. It was found that after being cocultured for $48 \mathrm{~h}$, the TDC $\mathrm{UCN}_{\mathrm{N}}$ group showed lower expressions of costimulatory molecules than the mature ones. FACS was significantly different between TDC $_{U C N}$ and $D_{\text {LPS }}$ with CD40 (1.7-fold) and CD80 (1.5-fold), which demonstrated that UCN induced TDCs could preserve the tolerant phenotype in an inflammatory environment (Figure 2D). Collectively, UCN was considered the most suitable one to induce TDCs.

Next, we attempted to construct a GO-based cell membrane targeted platform for the purpose of promoting the UCN delivery efficiency. UCN labeled by Cy 5 incubated and physically adsorbed on to excessive S-GO or L-GO. Results of fluorescence imaging showed that the conjugation efficiency of UCN with S-GO was $(89.57 \pm 1.53) \%$, compared with $(86.33 \pm 2.01) \%$ with L-GO (Figure 2E). The Fourier Transform Infrared (FT-IR) spectrometer was used to characterize the UCN@GO complex. A notable absorption peak of the peptide bond was observed compared to S-GO or L-GO alone (Figure 2F), which demonstrated the neuropeptide UCN was successfully conjugated with GO nanosheets. Flow cytometry was performed to detect the survival rate of DCs after incubation with different concentrations of UCN@GO complex for 48h. The survival rates of DCs varied significantly in a dose-dependent manner according to Figure 2G, which was why $15.6 \mu \mathrm{g} / \mathrm{ml}$ was chosen as the working concentration, for the corresponding survival rates in UCN-S and UCN-L group were both above $90 \%(90.02 \pm 2.95 \%$, $90.10 \pm 1.98 \%)$.

\section{GO as nanocarriers for UCN delivery and TDCs generation}

After being established for UCN, the GO delivery system was further evaluated in terms of TDCs generation. After incubation with UCN@GO for 48h, imDCs and the supernatant were collected separately. DCs were gated as CD11C+ population, CD40/80/86 showed no difference after incubation with free GO or UCN@GO (Supplementary Figure 5). ELISA measurements indicated that proinflammatory cytokines 
did not change significantly compared with imDCs after incubation with free GO or UCN@GO (Supplementary Figure 6). Further, the co-cultured DCs were added to CFSE labeled CD8+ T cells at a ratio of 1:4 for $48 \mathrm{~h}$. T cell proliferation rates of $\mathrm{DC}_{\mathrm{L}-\mathrm{GO}}$ and $\mathrm{DC} \mathrm{C}_{\mathrm{UCN} @ \mathrm{~L}-\mathrm{GO}}$ were $9.9 \%$ and $7.87 \%$, separately, compared with $10.94 \%$ of imDCs (Supplementary Figure 7). Therefore, we confirmed that neither free GO nor UCN@GO had any positive influence on DC maturity, which facilitated TDCs induction.

To simulate the environment where GVHD occurs, UCN@GO pretreated TDCs were further challenged in an inflammatory microenvironment induced by low-dose LPS. As a member of the CRF family, UCN mainly binds to CRHR2 expressed on cell membrane and affects inflammation response. CRHR2, a G protein-coupled receptors, is mainly expressed on cell membrane. BioGPS, a gene annotation portal, can provide complete gene information, including patterns of gene expressions in different cells or tissues. Data from BioGPS showed that CRHR2 expressions in the immune system, like BDCA4+ DCs, CD19+ B cells, CD8+ T cells, CD56+NK cells and CD14+ monocytes, is under median / were below the median value (Supplementary Figure 8). It was speculated that the low abundance of CRHR2 expressions on DC membrane might be the key to restricting the efficiency of TDCs induction. Therefore, improving the enrichment of UCN on the cell surface and promoting the binding efficacy of UCN and CRHR2 seemed to be an alternative solution to the limited production of TDCs.

We went on to explore whether GO loading would benefit the recognition of UCN by CRHR2 as expected. UCN was labeled with 5-TAMER and coupled with GOs, respectively. After DC incubation, FACS results indicated that UCN@L-GO tended to be adhered to the cytomembrane compared with UCN@S-GO or free UCN (29.3\% verse $20.0 \%$ and $14.8 \%$ ) (Figure 3A). 5-TAMER labeled UCN was further pre-conjugated with FITC-GOs, while DCs were stained with Cell Tracker ${ }^{\mathrm{TM}}$ Blue. Confocal imaging observation showed that most of the UCNs could be internalized in DCs cytoplasm without any GO carrier, which might fail to bind to the membrane receptor CRHR2. UCN@S-GO appeared to be in the cell cytoplasm, while UCN@L-GOs were mostly located on the surface of the cell membrane (Figure 3B), which conformed with the manner in which the carrier GO alone interacted. These results suggested that UCN@S-GO experienced a normal endocytosis process during incubation with DCs, while the larger lateral diameter of L-GO might probably benefit the concentration of UCN molecules on plasma membrane and the binding event with its receptor. The deduction was further verified by the co-location results of UCN and CRHR2 observed by the laser confocal (Supplementary Figure 9).

The downstream signaling molecular expressions were further examined by Western blot to explore the mechanism of TDC generation. Results showed that the presence of LPS had little influence on PKA C or CREB phosphorylation, so did single S-GO or L-GO treatment. UCN and UCN@GOs complex exhibited a similar increase of PKA C phosphorylation to $\mathrm{DC}_{\mathrm{LPS}}$. UCN incubation elicited more phosphorylation of the downstream CREB (1.81-fold), which indicated that UCN could effectively mediate TDCs production. And higher expressions of p-CREB (3.07-fold or 3.49-fold) were detected in the UCN@S-GO or UCN@L-GO group, suggesting that the enrichment of UCN on DC membrane, which resulted from GO coupling, could trigger cAMP/PKA pathway activation more effectively (Figure 3C and 3D). The above results served as 
evidence that GO had positive effects on the targeted delivery of UCN and promoted the combination of the ligand and receptor on the plasma membrane of DCs.

Once the CAMP/PKA/CREB signal pathway was activated, the CBP (CREB binding protein, CBP) molecules were translocated into the nucleus and further activated NF-KB, resulting in a decrease of proinflammatory cytokine expressions and co-stimulatory molecular levels of DCs. Thus, the levels of cytokine secretion and CD40/80/86 phenotype of TDCs were examined after LPS challenge. It was observed that the concentration of IL-12 $\mathrm{p}^{70}$ in the supernatant showed no significant difference in S-GO or L-GO treated groups compared with mature DCs. The concentration of IL-12 $\mathrm{p}^{70}$ decreased to $54.85 \pm 4.99$ pg/mL after UCN@S-GO pretreatment, and to $50.49 \pm 0.52$ pg/mL after UCN@L-GO incubation, which was significantly lower than in the free UCN group $(60.06 \pm 3.91 \mathrm{pg} / \mathrm{mL})$ and mature DC group $(85.97 \pm 2.19 \mathrm{pg} / \mathrm{mL})$. The amounts of IL-1 $\beta$, IL-6 and TNF-a secreted by DCs co-cultured with UCN@S-GO and UCN@L-GO also showed notable decreases compared to the free UCN group. These inflammatory cytokine levels demonstrated that the UCN-GO complex was more efficient in inducing TDCs than free UCN treatment (Figure 3E). Although S-GO or L-GO had little influence on expressions of costimulatory molecules, S-GO enhanced the efficiency of UCN in inhibiting the expression of surface costimulatory molecules CD40 (1.2-fold) and CD80 (1.3-fold) according to FACS results, compared with 1.5-fold, 1.7fold, and 1.1-fold after UCN@L-GO pretreatment, which exhibited a notable decrease in DCs maturity (Figure 3F). Given the positive role that GO played in promoting ligand and receptor combination and delivery of UCN, it could be speculated that the UCN@GO process enhanced UCN's ability to induce TDCs. Furthermore, it was obvious that UCN@L-GO outperformed UCN@S-GO in TDC generation. Thus, UCN@L$\mathrm{GO}$ was chosen as an inducer of TDCs for follow-up experiments.

\section{TDC $\mathrm{UCN} @ \mathrm{~L}-\mathrm{GO}_{\mathrm{O}}$ effectively deleted cytotoxic T cells and generated Tregs.}

Then, the T cell subtype and function were detected after co-culture with TDC $\mathrm{UCN}_{\mathrm{U}}$ or TDC $\mathrm{UCN}_{\mathrm{CL}-\mathrm{GO}}$. CD8+ T cells isolated from Balb/c mice were labeled with CFSE and incubated with different TDCs at the ratio of 4:1. After $48 \mathrm{~h}$ of incubation, FACS results showed that both TDC $\mathrm{UCN}_{\mathrm{CN}}$ and TDC $\mathrm{UCN}_{\mathrm{UL}-\mathrm{GO}}$ exhibited robust inhibition of cytotoxic T cell proliferation caused by LPS with a decrease of $39.4 \%$ and $25.1 \%$, respectively (Figure 4A). Next, the cytotoxic function of CD8+ T cells was evaluated by CD44, CD69 and CD107a. FACS demonstrated that CD8+ T cells lost their cytotoxic functions after incubation with TDC $\mathrm{UCN}_{\mathrm{N}}$ or TDC $_{U C N @ L-G O}$, for TDC $\mathrm{UCN}_{\mathrm{UL}-G O}$ suppressed the CD44/69/107a expression with a decrease of 79\%, 75\% and $64 \%$ compared with mature DC, respectively (Figure 4B).

According to primary research, despite the inhibition of CD8+ T cells through PD-L1 expression ${ }^{6}$, TDCs also mediated CD80/86 combination with CTLA4, leading to Treg generation. The proportion of Treg cells was further investigated in the population of CD4+ T cells. Notably, more CD4+ T cells obtained Treg phenotype after TDC $\mathrm{UCN}_{\mathrm{N}}$ or TDC $\mathrm{UCN}_{\mathrm{CL}-\mathrm{GO}}$ pretreatment, which was characterized by a remarkably increase in CD25 (1.38-fold and 1.63-fold) and CTLA4 (3.42-fold and 4.18-fold) compared with mature DCs (Figure 
4C). Collectively, these results indicated that UCN@L-GO complex was a promising candidate in TDC generation that led to anergy of cytotoxic $T$ cells and Treg expansion.

\section{UCN@L-GO complex triggered TDCs cytoskeleton rearrangement to promote the homing ability in vivo}

Another important feature of TDCs is the ability to migrate, for they have to home to lymph nodes to produce a notable influence. Despite the lateral size, another important discrepancy in physicochemical property between L-GO and S-GO is the content of carbon free radicals. According to electron paramagnetic resonance (EPR) results, more carbon free radicals would be measured on the surface of LGO compared to S-GO, which can be remarkably blocked by FBS (Supplementary Figure 10). Thus, it stands to reason to expect more ROS production in the $\mathrm{DC}_{\mathrm{L}-\mathrm{GO}}$ or TDC $\mathrm{UCN}_{\mathrm{UL}} \mathrm{L}-\mathrm{GO}$ co-incubation group, which has been further verified by FACS analysis as shown in Figure 5A. This indicated that ROS was accumulated during interactions between GOs and plasma membrane. Moreover, the expression of CCR7 on DC membrane showed the same tendency as ROS after co-incubation (Figure 5B). Collectively, we deduced that the adherence of L-GO to DC membrane was crucial to the activation of Rho A/ROCK pathway which existed in the downstream of ROS and participated in cell migration. Thus, the phosphorylation of Rho A/ROCK signals was further evaluated. L-GO amplified the phosphorylated levels of ROCK and MLC of DCs by 2.04-fold and 2.40-fold compared to the control DC. Although free UCN significantly suppressed p-ROCK and p-MLC, L-GO binding exhibited robust reversal (Figure 5C\&D). Because of the remarkable changes in p-ROCK and p-MLC, L-GO was considered an effective initiator of cytoskeleton rearrangement. Simultaneously, we observed a significant decrease in p-IKBa and p-P65 in $T_{U C N}$ and TDC $C_{U C N @ L-G O}$ groups, suggesting that TDCs remained the tolerant phenotype even in the presence of LPS.

Since cytoskeletal is the executor of cell migration, the rearrangement of DC cytoskeleton was observed by immunofluorescence (IF) in advance.

To simulate the inflammatory microenvironment in the process of GVHD, low-dose LPS was added to the co-culture system as a stimulus to TDC challenge. Obviously, L-GO materials had positive effect on the rearrangement of DC cytoskeleton characterized by increased expressions of F-Actin and $\beta$-tubulin. In contrast, free UCN showed a notable decrease, which might have been brought about by inflammatory inhibition. However, after L-GO binding, TDC $\mathrm{UCN}_{\mathrm{CL}-\mathrm{GO}}$ displayed remarkable restoration of F-Actin and $\beta$ tubulin expressions. To be more specific, the fluorescence intensity of F-Actin and $\beta$-tubulin in the TDC $_{U C N @ L-G O}$ group was 2.31-fold and 1.42-fold higher than that of $T_{D C} C_{U C N}$, respectively (Figure 5E\&F). In the absence of LPS, a similar tendency was also observed as shown in Supplementary Figure 11. Thus, the strong migration ability of TDCs induced by UCN@L-GO complex was expected. Combined with the Western blotting results in Figure 5C, it could be concluded that the GO-based cytomembrane-philic delivery platform participated in cytoskeletal rearrangement via ROS generation and ROCK phosphorylation. 
Living cell imaging was carried out to track and analyze a time-laps cell migration pathway and length. After $6 \mathrm{~h}$ of observation, it was found that the L-GO had a stronger ability to induce DCs migration and longer displacement in contrast to UCN (Figure 5G). And the complex of UCN@L-GO could significantly restore the migration ability of TDCs impaired by UCN. L-GOs treatment also accelerated cell migration velocity. Unlike the disordered movements of the control DCs, DCs incubated with UCN@L-GO showed faster track velocity with a dominant motile mode of classical amoebic movement, which displayed a more efficacy migration mode (Figure $5 \mathrm{H}$ ).

Furthermore, we employed the "footpad injection model" to confirm the ability of DCs to home to lymph nodes (LNs) after being co-cultured with UCN and UCN@L-GO. Mice were observed at time points of $24 \mathrm{~h}$, $48 \mathrm{~h}$ and $72 \mathrm{~h}$ after footpad inoculation of Fluc ${ }^{+}$DCs using the fluorescence imaging system. The migration rate of DCs was measured by the ratio of fluorescence signals in PLNs (popliteal lymph nodes, PLNs) to the signals remaining on the foot pad. LPS was employed as a positive control. After L-GO treatment, DCs acquired a much stronger ability to home to PLNs than immature DCs, while free UCN treatment contributed to promotion. TDC $\mathrm{UCN}_{\mathrm{CL}-\mathrm{GO}}$ exhibited a more significant migration rate than TDC $_{U C N}$. Comparisons between the groups demonstrated that in the TDC $\mathrm{UCN}_{\mathrm{LL}-\mathrm{GO}}$ group, the ratio of DCs migrating to PLNs at $24 \mathrm{~h}, 48 \mathrm{~h}$ and $72 \mathrm{~h}$ were respectively $17.75 \pm 4.11,19.75 \pm 5.09$ and $25.24 \pm 5.07$, which was the highest among these groups. (Figure 6A\&B).

We also investigated the distribution of Fluc ${ }^{+} \mathrm{DCs}$ after intravenous administration in vivo. Fluorescence signals were detected using the fluorescence imaging system at $4 \mathrm{~h}, 24 \mathrm{~h}$ and $48 \mathrm{~h}$ post injection. Results showed that the entire fluorescence intensities were hardly different between these groups at different time points (Figure 6C\&D). Organs were collected at $72 \mathrm{~h}$ post injection and imaged by the fluorescence system. The LPS group exhibited much stronger migration to lymphoid tissue than the control did. Since

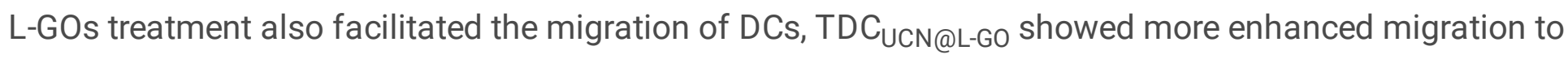
lymphoid tissues than free UCN treatment, as was illustrated by analysis of fluorescence intensities (Figure 6E). Statistical analysis also demonstrated that UCN@L-GO treatment accelerated cell migration to lymphoid tissues more significantly than free UCN co-cultured ones (Figure 6F).

Moreover, the isolated organs were separated into two groups, solid organs and lymphoid ones. Results confirmed that TDC $C_{U C N @ L-G O}$ exhibited stronger migration capacity to spleen and lymph nodes than $\mathrm{TDC}_{\mathrm{UCN}}$, which probably benefitted the interaction and regulation with T cells (Figure 6G).

\section{UCN@L-GO treated TDCs regulated the phenotype of donor T cells and protected recipients from lethal GVHD}

An acute GVHD mouse model was established to help find out whether the TDC UCN@L-GO could notably inhibit cytotoxic T cell proliferation or generate adequate Treg cells in GVHD relief.

To determine the amount of L-GOs that model mice would be exposed to or caused by DCs immunotherapy, FITC labelled L-GO or UCN@L-GO were incubated with DCs for 48 h. The supernatant 
and cells were collected separately for fluorescence detection. Results in Supplementary Figure 12 illustrated that the residual L-GOs that adhered onto the cytomembrane of DCs were less than $30 \%$ of the incubated amount with the excessive L-GO or UCN@L-GO mainly in the cell culture medium. A widely accepted visualized GVHD murine model was established by infusion of a cell mixture composed by $T$ cell-deleted allogenic bone marrow cells and Fluc+ $T$ cells. And the entire fluorescence intensity could represent the proliferation level of T cells in vivo. The group labeled as "BM+T" meant no successive immunotherapy treatment. After transplantation, $4 \times 10^{6} \mathrm{DCs}, \mathrm{DC}_{\mathrm{L}-\mathrm{GO}}, \mathrm{TDC}_{\mathrm{UCN}}$ and TDC $\mathrm{UCN}_{\mathrm{L}-\mathrm{GO}}$ were infused intravenously respectively (Supplementary Figure 13). On the 14th day post transplantation, imaging analysis showed the "BM+T" group had the highest fluorescence intensity, which indicated massive activation and acute proliferation of $\mathrm{T}$ cells. Injection of $\mathrm{DC}_{\mathrm{L}-\mathrm{GO}}$ resulted in little difference compared with the DCs group, while TDC $\mathrm{UCN}_{\mathrm{N}}$ displayed a 2.37-fold decrease in fluorescence intensity. Furthermore, the TDC $\mathrm{UCN}_{\mathrm{UL}-\mathrm{GO}}$ group showed 2.8-fold reduction compared with the TDC $\mathrm{UCN}_{\mathrm{C}}$ group, suggesting the positive effect of L-GOs binding in inhibiting T cell proliferation (Figure 7A\&B). The LNs, lung, thymus, spleen, pancreas, liver, intestine and kidney were isolated and imaged. Results showed that the $\mathrm{BM}+\mathrm{T}$ group had extensive $\mathrm{T}$ cell proliferation in multiple tissues, especially in the spleen, mesenteric lymph nodes (MLNs), thymus and small intestine (SI), which indicated rapid progress of GVHD. The infusion of control DCs or $\mathrm{DC}_{\mathrm{L}-\mathrm{GO}}$ displayed relief to a certain extent. As expected, TDC $\mathrm{UCN}_{\mathrm{N}}$ and TDC $\mathrm{C}_{\mathrm{UCN} @ \mathrm{~L}-}$ Go groups both exhibited a significant decrease in organ fluorescence intensities, especially the latter, which induced apoptotic deletion of donor CD8+ T cells (Figure 7C\&D). ELISA was performed to analyze the cytokine levels in serum. It was found that after transplantation, such proinflammatory cytokines as IL-1 $\beta$, IL- 6 and MCP- 1 were robustly increased, while the anti-inflammatory cytokines of IL-10 and TGF- $\beta$ both significantly decreased compared to the irradiation control, which was a sign of the explosive inflammation in vivo. Infusion of control $D C s$ and $D_{\mathrm{L}_{-G O}}$ had slight effect on cytokine secretion, while $\mathrm{TDC}_{\mathrm{UCN}}$ and TDC $\mathrm{UCN} @ \mathrm{~L}-\mathrm{GO}_{\mathrm{induced}}$ an obvious decrease in these proinflammatory cytokines and a notable increase in the anti-inflammatory ones. (Figure 7E).

TDCs also play non-redundant roles in promoting expansion and function of Tregs. As primary studies have showed, TDCs can induce T cell tolerance broadly via two categories: intrinsic and extrinsic. TDCs produce PD-L1 and CD80/86, which bind to PD-1 and CTLA-4 respectively, leading to the deletion of cytotoxic $T$ cells and expansion of Tregs that can obstruct the proliferation and survival of those activated T cells. Moreover, TDCs can suppress the antigen-reactive T cells and promote Treg generation through IDO production ${ }^{6}$. We further isolated the MLNs and spleens to evaluate the proportion of Tregs. FACS showed that the proportion of Treg increased dramatically both in the spleen and MLNs after TDC treatment. According to the FACS results, TDC $U$ UCN@L-GO injection mediated 1.67-fold and 9.23-fold increases of CD4+CD154+ and $66.7 \%$ and $73.7 \%$ decreases of CD4+CTLA4+ in the spleen and MLNs separately, compared to BM+T group, which both corresponded to the immune suppressive T cells (Figure 7F).

Cytotoxic T cell apoptotic deletion and Tregs generated by TDCs both played a vital role in GVHD relief by mitigating tissue damage. Hematoxylin and eosin (HE) staining of the involved organs confirmed the 
positive effect. The BM+T group was attacked by the donor T cells in multiple organs and had massive lymphocyte infiltration, which were symptoms of severe GVHD. DCs and DC $C_{\mathrm{L}-G \mathrm{O}}$ infusion both showed limited remission, while TDC $\mathrm{UCN}_{\mathrm{U}}$ and TDC $\mathrm{UCN}_{\mathrm{UL}-\mathrm{GO}}$ displayed notable improvement of multiple organs involved such as the spleen, liver, MLN, thymus and SI (Figure 7G, Supplementary Figure 14), especially the latter. Also, mice which received TDCs treatment, especially those in TDC $\mathrm{UCN}_{\mathrm{UL}-\mathrm{GO}}$ group, showed remarkable abrogation in symptoms caused by severe GVHD, including hunched posture, skin and ear inerythema, alopecia and weight loss. Altogether, these results indicated that UCN@L-GO treatment was extremely efficient for TDC generation, which was probably the key to altering $T$ cell subtype and the resultant GVHD relief in vivo.

\section{Discussion}

Lymph node-resident immune cells are usually chosen as the targeting subpopulation for immunoregulation of allo-response in allogeneic bone marrow transplantation (BMT), organ transplantation rejection or autoimmune disease treatment. However, it is generally difficult to ensure the drug can reach the targeted cells or locations with the effective concentration because of the limitation of toxicity or low solubility, even under accurate control in drug delivery. Alternatively, local delivery methods that can generate regulatory DCs or T cells ex vivo under appropriate conditions could serve as a second solution. When the nanotechnology-based strategy was introduced for the delivery of immunomediators, the advantages of adoptive immunotherapy were magnified to some extent, for this approach required a smaller quantity of nano-carriers or drugs that entered the circulatory system, increasing the chance of favorable biocompatibility. To be more specific, only a small amount of GO nanosheets $(0.8 \mu \mathrm{g}$ per mouse, less than $30 \%$ of the incubation amount) would enter the organism along with TDCs infusion, which increased the likelihood of transformation from bench to bedside.

Herein, the bone marrow derived dendritic cells from the recipient were selected as the source of TDCs, given that host APCs, which were major histocompatibility complex (MHC)-matched with host antigens, were reported to provide more effective protection than donor DCs, which were host-mismatched ones, from lethal GVHD, with a remarkable increase of recipient survival ${ }^{30,31}$. Meanwhile, preclinical data of murine models showed that donor DCs, activated by donor engraft, secreted rising levels of inflammatory cytokines which exacerbated the lethal feed-forward GVHD cascade ${ }^{32,33}$.

UCN, one of neuropeptides produced by different cells and tissues, is associated with distinct physiological functions. It was activated in response to inflammation, injury and pathogen clearance. UCN binds to CRHR2, a G-protein-coupled receptor, and is expressed in different immunocompetent cells. CRHR2 is coupled to the activation of the CAMP/PKA/CREB pathway, which is related to downregulation of several transduction pathways associated with transcription of inflammatory mediators, like proinflammatory cytokines secretion and co-stimulatory molecule expression. However, it is worth noting that CRHR2 has a relatively low abundance on DC membrane, which is the major barrier to TDCs induction by UCN treatment. The excellent performance of the UCN@L-GO as an immunomodulator in 
inducing TDCs is due to the unique manner in which they interact with DCs, namely adhesion or adsorption onto the plasma membrane, which was known as the cytomembrane-philic property in the current work. Receptor-ligand interactions, which are crucial to various pathophysiological processes, proved to be a kinetic equilibrium of combination and dissociation. Thus, regulation of the dynamic process might improve the integration efficacy of receptors and ligands. As has been verified in the interaction model of a biotin-labeled RBCs system, the presence of L-GOs and their preferred location at the edges of plasma membrane contributed effectively to the encountering and binding of biotin and avidin, especially when the surface abundance of biotin was relatively low (corresponding to the biotin dosage of 1,2 , and $4 \mu \mathrm{g}$ ), much like the expression level of cytomembrane receptors of UCN, namely CRHR2, on the surface of DCs (Supplementary Figure 8). This led to the conclusion that L-GO could enormously enhance TDC generation through non-specific UCN delivery and promoted UCN-CRHR2 binding.

The migration toward draining lymph nodes is critical to DCs in their induction and maintenance of adaptive immunity. Generally speaking, their high mobility is readily observed under in $\square$ ammatory or activated conditions. In other words, tolerant or other mediate-phenotype DCs are not usually capable of homing to secondary lymph-nodes because of the low expression level of CCR7 and the non-organized cytoskeletons, which are often accompanied by low surface costimulatory molecules and proinflammatory cytokine, such as IL-6, IL-12 $p^{70}, \mathrm{IL}-1 \beta$ and TNF-a (Figure 3E). Traditional systemic use of immunosuppressive drugs is facing such challenges, for it will impair immune function and cause side effects. According to previous research, TDCs generated by rapamycine showed poor performance in cell movements ${ }^{34}$. In order to obtain TDCs with enhanced homing ability of lymph nodes, other researchers have attempted to employ adenovirus as a vector to overexpress CCR7 on DCs to preserve immune tolerance simultaneously. But the limited efficacy and security concerns stand in the way. In the case of UCN@L-GO pulsed DCs, it was a pleasant surprise to find the high expression level of CCR7 (Figure 5B) as well as the promotion of the dynamic reconstruction of F-actin and $\beta$-tubulin in cytoskeleton (Figure 5E). This could be attributed to the abundance of carbon free radicals in L-GO in comparison with their small counterpart, which led to the more production of reaction oxygen species (ROS) and Rho A/ROCK pathway activation as shown in Figure 5A\&C. Such a conclusion is also supported by literature that says the mechanism by which exogenous $\mathrm{H}_{2} \mathrm{O}_{2}$ promotes migration is due to the up-regulation of CCR7 receptor on the surface of DCs and activation of the downstream PI3K/Akt and NF-KB pathways. Our results also demonstrated notable phosphorylation in both IKb/NF-kb and Rho A/ROCK pathway after LGO or UCN@L-GO incubation ${ }^{35}$. As a result, strengthened homing ability of the current TDCs to secondary lymphoid tissues was also observed (Figure 6). This might be one of the special advantages brought about by nano-carriers, or more precisely, by GO nanosheets over molecular biology-based methods.

Engineering nanomaterials, such as PLGA ${ }^{36-38}$, have emerged as newly reported carriers for immune modulatory agent delivery in transplantation rejection treatment, making it necessary to address the overall advantages of employing GO as carrier over other nanoparticles. The high surface area of GO allows the accumulation of immunomodulators through coupling, and thus the high density of 
neuropeptide appears in the adjacent region of their receptor. Moreover, unlike other nanomaterials, largesized GO nanosheets mediated the interaction related to non-specific adhesion or adsorption onto the surface of cell plasma membrane, which is by no means passive phagocytosis. As it is generally believed that phagocytosis would promote the differentiation from immature DCs to a more mature phenotype, this distinctive property of GO meets the particular requirements for the generation of tolerant DCs. Moreover, as has been discussed previously, exogenous carbon free radicals from L-GO might probably be the main contributor to the overexpression of CCR7 and rearrangement of cytoskeleton, which might account for the promoted homing ability of lymph nodes and the inhibition of effector donor $\mathrm{T}$ cells.

\section{Conclusion}

In this study, we designed novel immune-inducer of TDCs composed of UCN and L-GO which were capable of facilitating TDCs generation, and the immunotherapy of TDCs remarkably inhibited cytotoxic T cell activation in acute GVHD. The overall process was schematically presented in Figure 8. Unlike their small counterparts, large-sized GOs proved to be cytomembrane-philic and effectively promote the recognition between UCN and its receptor CRHR2 expressed on the membrane. Moreover, L-GOs also contributed to the organization of cytoskeleton via Rho A/ROCK pathway and elevation of CCR7 levels, which could enhance cell mobility and homing efficacy even under the tolerant phenotype. Adoptive immunotherapy of TDC UCN@L-GO exhibited excellent efficiency with promoted migration to lymph nodes, remarkable inhibition of cytotoxic $T$ cell proliferation and statistically significant generation of Tregs, thus preventing the progress of GVHD. Finally, our study provides an efficient TDC inducer initiated by UCN@LGO, which can be a promising candidate in immunotherapy of GVHD.

\section{Declarations}

\section{Acknowledgements}

This study was supported in part by the National Nature Science Foundation of China (81770196, 81970166, 81903624 and 81701583).

\section{References}

1. Hippen, K. L., Aguilar, E. G., Rhee, S. Y., Bolivar-Wagers, S. \& Blazar, B. R. Distinct Regulatory and Effector T Cell Metabolic Demands during Graft-Versus-Host Disease. Trends Immuno/ 41, 77-91, doi:10.1016/j.it.2019.11.005 (2020).

2. Zhang, L., Yu, J. \& Wei, W. Advance in Targeted Immunotherapy for Graft-Versus-Host Disease. Front Immuno/ 9, 1087, doi:10.3389/fimmu.2018.01087 (2018).

3. Godoy, J. A. P. et al. Clinical Translation of Mesenchymal Stromal Cell Therapy for Graft Versus Host Disease. Frontiers in cell and developmental biology 7, 255, doi:10.3389/fcell.2019.00255 (2019). 
4. Blazar, B. R., Murphy, W. J. \& Abedi, M. Advances in graft-versus-host disease biology and therapy. Nature reviews. Immunology 12, 443-458, doi:10.1038/nri3212 (2012).

5. Miyamoto, T. et al. Comparison of cyclosporine and tacrolimus combined with mycophenolate mofetil in prophylaxis for graft-versus-host disease after reduced-intensity umbilical cord blood transplantation. International journal of hematology 105, 92-99, doi:10.1007/s12185-016-2093-0 (2017).

6. Yu, H., Tian, Y., Wang, Y., Mineishi, S. \& Zhang, Y. Dendritic Cell Regulation of Graft-Vs.-Host Disease: Immunostimulation and Tolerance. Front Immunol 10, 93, doi:10.3389/fimmu.2019.00093 (2019).

7. Choi, S. W. \& Reddy, P. Current and emerging strategies for the prevention of graft-versus-host disease. Nature reviews. Clinical oncology 11, 536-547, doi:10.1038/nrclinonc.2014.102 (2014).

8. Audiger, C., Rahman, M. J., Yun, T. J., Tarbell, K. V. \& Lesage, S. The Importance of Dendritic Cells in Maintaining Immune Tolerance. Journal of immunology 198, 2223-2231, doi:10.4049/jimmunol.1601629 (2017).

9. Chung, C. Y., Ysebaert, D., Berneman, Z. N. \& Cools, N. Dendritic cells: cellular mediators for immunological tolerance. Clinical \& developmental immunology 2013, 972865, doi:10.1155/2013/972865 (2013).

10. Devi, K. S. \& Anandasabapathy, N. The origin of DCs and capacity for immunologic tolerance in central and peripheral tissues. Seminars in immunopathology 39, 137-152, doi:10.1007/s00281016-0602-0 (2017).

11. MacDonald, K. P., Shlomchik, W. D. \& Reddy, P. Biology of graft-versus-host responses: recent insights. Biology of blood and marrow transplantation: journal of the American Society for Blood and Marrow Transplantation 19, S10-14, doi:10.1016/j.bbmt.2012.11.005 (2013).

12. Ganguly, D., Haak, S., Sisirak, V. \& Reizis, B. The role of dendritic cells in autoimmunity. Nature reviews. Immunology 13, 566-577, doi:10.1038/nri3477 (2013).

13. Price, J. D. \& Tarbell, K. V. The Role of Dendritic Cell Subsets and Innate Immunity in the Pathogenesis of Type 1 Diabetes and Other Autoimmune Diseases. Frontiers in immunology 6, 288, doi:10.3389/fimmu.2015.00288 (2015).

14. Reizis, B., Colonna, M., Trinchieri, G., Barrat, F. \& Gilliet, M. Plasmacytoid dendritic cells: one-trick ponies or workhorses of the immune system? Nature reviews. Immunology 11, 558-565, doi:10.1038/nri3027 (2011).

15. Delgado, M., Chorny, A., Ganea, D. \& Gonzalez-Rey, E. Vasoactive intestinal polypeptide induces regulatory dendritic cells that prevent acute graft versus host disease and leukemia relapse after bone marrow transplantation. Annals of the New York Academy of Sciences 1070, 226-232, doi:10.1196/annals.1317.019 (2006).

16. Garcia-Gonzalez, P., Ubilla-Olguin, G., Catalan, D., Schinnerling, K. \& Aguillon, J. C. Tolerogenic dendritic cells for reprogramming of lymphocyte responses in autoimmune diseases. Autoimmunity reviews 15, 1071-1080, doi:10.1016/j.autrev.2016.07.032 (2016). 
17. Menges, M. et al. Repetitive injections of dendritic cells matured with tumor necrosis factor alpha induce antigen-specific protection of mice from autoimmunity. The Journal of experimental medicine 195, 15-21, doi:10.1084/jem.20011341 (2002).

18. Yamazaki, S. et al. Dendritic cells are specialized accessory cells along with TGF- for the differentiation of Foxp3+CD4+ regulatory T cells from peripheral Foxp3 precursors. Blood 110, 4293-4302, doi:10.1182/blood-2007-05-088831 (2007).

19. Gonzalez-Rey, E., Chorny, A. \& Delgado, M. Regulation of immune tolerance by anti-inflammatory neuropeptides. Nat Rev Immunol 7, 52-63, doi:10.1038/nri1984 (2007).

20. Pan, W. \& Kastin, A. J. Urocortin and the brain. Progress in neurobiology $84,148-156$, doi:10.1016/j.pneurobio.2007.10.008 (2008).

21. Oki, Y. \& Sasano, H. Localization and physiological roles of urocortin. Peptides $25,1745-1749$, doi:10.1016/j.peptides.2004.06.023 (2004).

22. Elsasser, T. H. \& Kahl, S. Adrenomedullin has multiple roles in disease stress: development and remission of the inflammatory response. Microscopy research and technique 57, 120-129, doi:10.1002/jemt.10058 (2002).

23. Gonzalez-Rey, E., Chorny, A., Fernandez-Martin, A., Ganea, D. \& Delgado, M. Vasoactive intestinal peptide generates human tolerogenic dendritic cells that induce CD4 and CD8 regulatory T cells. Blood 107, 3632-3638, doi:10.1182/blood-2005-11-4497 (2006).

24. Bao, H. et al. Chitosan-functionalized graphene oxide as a nanocarrier for drug and gene delivery. Small 7, 1569-1578, doi:10.1002/smll.201100191 (2011).

25. Jung, J. H., Cheon, D. S., Liu, F., Lee, K. B. \& Seo, T. S. A graphene oxide based immuno-biosensor for pathogen detection. Angewandte Chemie 49, 5708-5711, doi:10.1002/anie.201001428 (2010).

26. Kanagavalli, P. \& Veerapandian, M. Opto-electrochemical functionality of Ru(II)-reinforced graphene oxide nanosheets for immunosensing of dengue virus non-structural 1 protein. Biosensors \& bioelectronics 150, 111878, doi:10.1016/j.bios.2019.111878 (2020).

27. Sun, X. et al. Nano-Graphene Oxide for Cellular Imaging and Drug Delivery. Nano research 1, 203212, doi:10.1007/s12274-008-8021-8 (2008).

28. Yang, K. et al. Graphene in mice: ultrahigh in vivo tumor uptake and efficient photothermal therapy. Nano letters 10, 3318-3323, doi:10.1021/nl100996u (2010).

29. Tkach, A. V. et al. Graphene oxide, but not fullerenes, targets immunoproteasomes and suppresses antigen presentation by dendritic cells. Smal/ 9, 1686-1690, doi:10.1002/smll.201201546 (2013).

30. Chorny, A., Gonzalez-Rey, E., Fernandez-Martin, A., Ganea, D. \& Delgado, M. Vasoactive intestinal peptide induces regulatory dendritic cells that prevent acute graft-versus-host disease while maintaining the graft-versus-tumor response. Blood 107, 3787-3794, doi:10.1182/blood-2005-114495 (2006).

31. Sato, K., Yamashita, N., Baba, M. \& Matsuyama, T. Modified myeloid dendritic cells act as regulatory dendritic cells to induce anergic and regulatory T cells. Blood 101, 3581-3589, doi:10.1182/blood2002-09-2712 (2003). 
32. Turner, B. E. et al. Reduced intensity conditioning for allogeneic hematopoietic stem-cell transplant determines the kinetics of acute graft-versus-host disease. Transplantation 86, 968-976, doi:10.1097/TP.0b013e3181874787 (2008).

33. Koyama, M. \& Hill, G. R. The primacy of gastrointestinal tract antigen-presenting cells in lethal graftversus-host disease. Blood 134, 2139-2148, doi:10.1182/blood.2019000823 (2019).

34. Reichardt, W. et al. Impact of mammalian target of rapamycin inhibition on lymphoid homing and tolerogenic function of nanoparticle-labeled dendritic cells following allogeneic hematopoietic cell transplantation. J Immunol 181, 4770-4779, doi:10.4049/jimmunol.181.7.4770 (2008).

35. Batal, I. et al. The mechanisms of up-regulation of dendritic cell activity by oxidative stress. $J$ Leukoc Bio/ 96, 283-293, doi:10.1189/jlb.3A0113-033RR (2014).

36. Liu, Q. et al. Use of Polymeric Nanoparticle Platform Targeting the Liver To Induce Treg-Mediated Antigen-Specific Immune Tolerance in a Pulmonary Allergen Sensitization Model. ACS Nano 13, 4778-4794, doi:10.1021/acsnano.9b01444 (2019).

37. Dane, K. Y. et al. Nano-sized drug-loaded micelles deliver payload to lymph node immune cells and prolong allograft survival. J Control Release 156, 154-160, doi:10.1016/j.jconrel.2011.08.009 (2011).

38. Pan, Q. et al. Corticosteroid-loaded biodegradable nanoparticles for prevention of corneal allograft rejection in rats. J Control Release 201, 32-40, doi:10.1016/j.jconrel.2015.01.009 (2015).

\section{Methods}

\section{Mice.}

Male wild-type C57BL/6J mice, 6-8 weeks, were obtained from the Academy of Military Medicine Science Animal Center. L2G85 (FVB) mice expressing Firefly luciferase (Fluc ${ }^{+}$) were backcrossed with C57BL/6J mice and used at stage N7 (L2G85.C57BL/6J). Fluc ${ }^{+}$BMDCs induced from L2G85.C57BL/6J mice were used for bioluminescence imaging. The mice were housed under SPF conditions at the National Beijing Center for Drug Safety Evaluation and Research (NBCDSER). All animal studies were conducted in compliance with the guidelines of National Institutes of Health Guide for the Care and Use of Laboratory Animals.

\section{Characterization of DCs}

DCs generated from bone marrow were cultured in complete RPMI 1640 medium. Flow cytometry was performed for phenotype detection of DCs. DCs were washed twice with PBS and stained with CD11C, CD40, CD80 and CD86 antibodies. After 15-min incubation, cells were washed with $1 \mathrm{~mL}$ PBS and suspended with $200 \mu \mathrm{L}$ binding buffer for fluorescence activated cell sorting (FACS). Data were collected and analyzed with CellQuest software. Isotype contrast of each antibody was used for gate control, and CD11c+ cells were defined as DCs. 


\section{Identification of GO location on cell membrane}

$2 \times 10^{6}$ Red blood cells (RBCs) were biotined with gradient S-NHS (Abcam. USA) and incubated for 60 min at $37^{\circ} \mathrm{C}$. The biotinalyzed-RBCs were washed and suspended with $1 \mathrm{~mL}$ PBS, GO pretreated PE-avidin (Biolegend, USA) was added to the biotinalized RBCs and incubated for 60 min in dark. Next, cells were washed again and suspended with $200 \mu \mathrm{L}$ binding buffer. PE positive rate and mean fluorescence intensity were analyzed by CellQuest software.

$2 \times 10^{6} \mathrm{DC}$ s were seeded in six-well plate and co-cultured with FITC-GO for $48 \mathrm{~h}$. After incubation, cells were fixed and stained with rhodamine. Laser confocal were employed to investigate GO location on DC membrane.

\section{GO and UCN conjugation.}

Neuropepitide was purchased from Jier Biochemical (Shanghai) Co., LTD, (amino acid sequence was provided in Table 1). $2 \times 10^{6} \mathrm{DCs}$ were cultured with complete RPMI 1640 medium and incubated with $10^{-5}$ $\mathrm{M}$ neuropeptide for $48 \mathrm{~h}$ in six-well plates. After incubation, cell medium and DCs were separately collected. Cells were labeled with CD11c, CD40/80/86 antibodies to character the maturity of DCs. Cell

medium were gathered for cytokine detection according to ELISA instructions, including IL-6, IL-12 $p^{70}$, IL$1 \beta$, and TNF-a.

CD8+ T cells derived from spleen were isolated with immunomagnetism and activated with ConA. Then, T cells were stained with carboxyfluoresceinsuccinimidyl amino ester (CFSE) and co-cultured with DCs pretreated with neuropeptide for $72 \mathrm{~h}$. After incubation, cells were collected and labeled with perCPCD8 antibody (Biolegend, USA). Flow cytometry was used for cytotoxic $T$ cell proliferation detection.

For preparation of UCN@GO complex, gradient GO and $10^{-5} \mathrm{M}$ UCN were suspended with $\mathrm{ddH}_{2} \mathrm{O}$ to $200 \mu \mathrm{l}$ final volume. The UCN@GO mixture was incubated for $30 \mathrm{~min}$ at a rotational speed of $200 \mathrm{rpm} / \mathrm{min}$, and stationary at room temperature for 2h. $2 \times 10^{6}$ DCs were incubated with UCN@GO complex at different concentrations for $48 \mathrm{~h}$. To detect cell apoptosis, cells were collected and washed with PBS. Next, binding buffer was used for cell suspend, Annexin-FITC and propidium iodide (PI) were added to DCs. After incubation, the ratio of apoptosis cells was analyzed with CellQuest software.

\section{Characteristic of tolerogenic DCs.}

To investigate the location of UCN@GO with DCs, laser confocal was used after co-culture for 48h. After incubation with FITC-GO and 5-TAMRA-UCN, cells were fixed and stained with Cell Tracker $^{\text {TM }}$ Blue CMAC (Invitrogen, USA).

After being co-cultured with UCN@GO, DCs and cell medium were collected separately. Next, cell costimulatory marker and cytokines were detected as described above.

\section{In vitro cell migration analysis}

Page 19/30 
DCs derived from GFP transgene mice were isolated and co-cultured with UCN@GO complex and timelapse cell migration observation was performed using a living cell imaging system (PerkinElmer, Massachusetts, USA). Then cell tracking data were analyzed by Velocity software including track length and track velocity.

Cytoskeleton rearrangement was measured after different treatments by immunofluorescence. Cells in each group were fixed and permeabilized before stained with anti-F-actin (Abcam, USA) and anti- $\beta$-tubulin (Abcam, USA) overnight. Next, cells were washed and conjugated with AF-488 and AF-594 IgG (Abcam, USA) for $2 h$ in dark, separately. The cells were then mounted using fluoroshield mounting medium with DAPI (Abcam) and observed under a laser confocal microscope (PerkinElmer, Massachusetts, USA).

\section{Bioluminescence imaging of DCs migration and distribution in vivo}

We investigated bioluminescence imaging with an IVIS system (PerkinElmer, Inc.) to reflect in vivo cell migration and distribution after different treatment. For DCs migration measurements, Fluc+ DCs were injected through footpad. Imaging data were collected and cell migration rate was calculated as the signal intensity ratio of the PLN to the entirety. For DCs distribution, Fluc+ DCs were intravenously injected to wild type C57BL/6J mice and observed at $4 \mathrm{~h}, 24 \mathrm{~h}, 48 \mathrm{~h}$ and $72 \mathrm{~h}$. Mice fur was depilated to improve light specific. Mice were scarified $72 \mathrm{~h}$ after injection, tissues were dissected and imaged for 1 min to obtain photographs.

\section{DCs treatment for allo-HSCT mouse model}

An Allo-HSCT mouse model was established as an acute GVHD model to investigate different DC treatments. DCs were pretreated with UCN@GO complex for 48h. Fluc+ lymphocytes were isolated from the spleen of FVB Fluc+ Balb/c mice. Wild type C57BL/6J mice were chosen as recipients which were depilated and received $8 \mathrm{~Gy} \mathrm{Co}^{60}$ irradiation. Fluc+ lymphocytes and DCs with different treatments were intravenously injected to the recipients while the activation of transplanted $T$ cells was reflected by the fluorescence intensity. Mice were sacrificed 14-day post injection, serum and tissues were collected for therapy efficacy evaluation. Tissues were dissected and imaged to monitor the activity of transplanted $T$ cells in each organ. Next, all tissues were fixed and sectioned for hematoxylin-eosin (HE) staining. Serum cytokines, like IL-1 $\beta$, IL-6, MCP-1, IL-10 and TGF- $\beta$, were measured by ELISA according to the instruction.

\section{Statistical analysis}

Data were analyzed using SPSS (Version 20.0). Dunnett's t-test or one-way ANOVA analysis of variance was used to analyze the normally distributed data. Non-parametric testing was used to analyze the nonnormally distributed data. $p<0.05$ was considered to indicate a significant difference.

\section{Table}

Table 1 is not available with this version. 


\section{Figures}

A

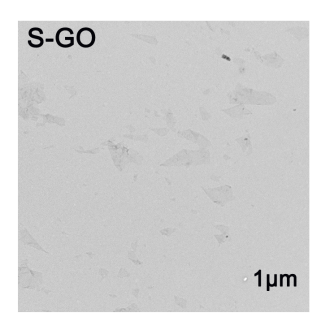

$\mathrm{C}$
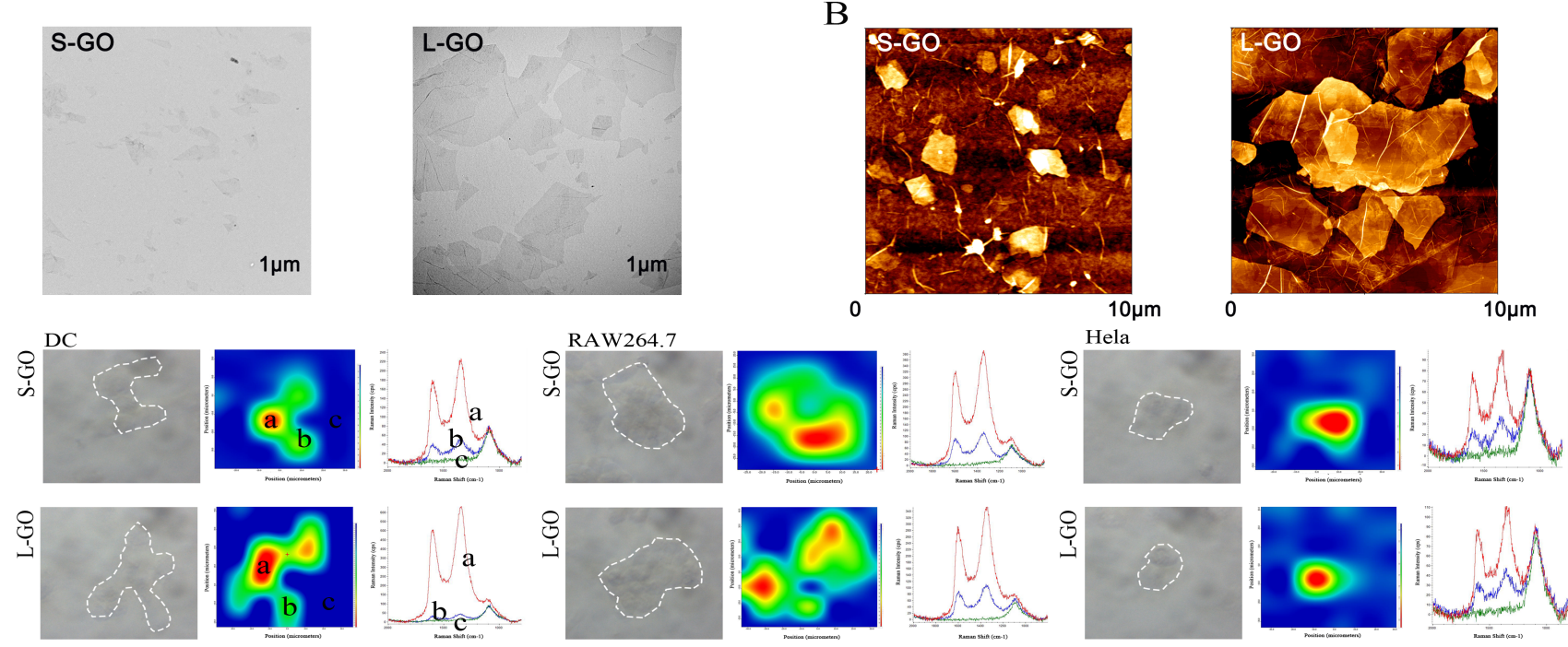

D
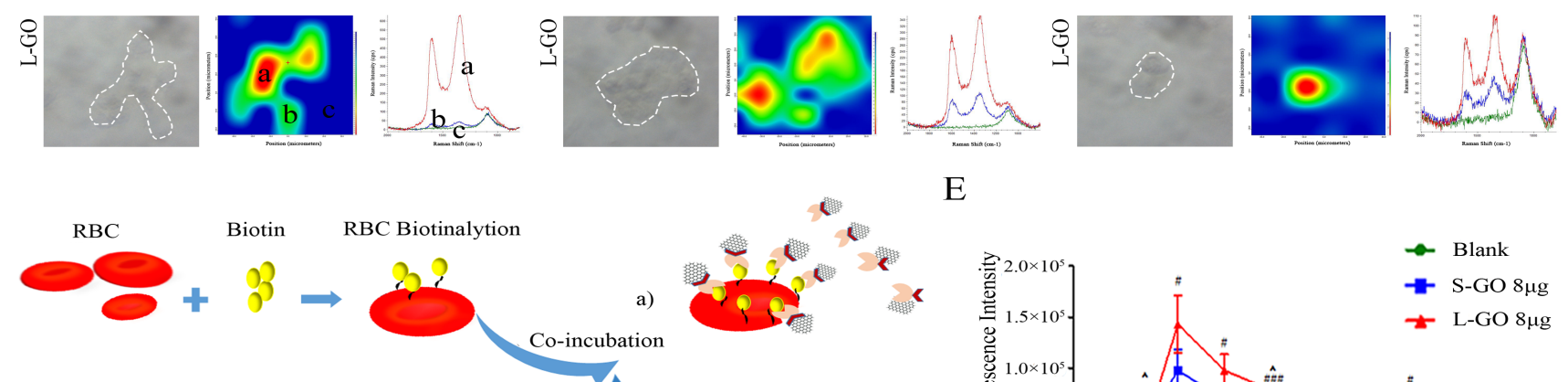

$\mathrm{E}$

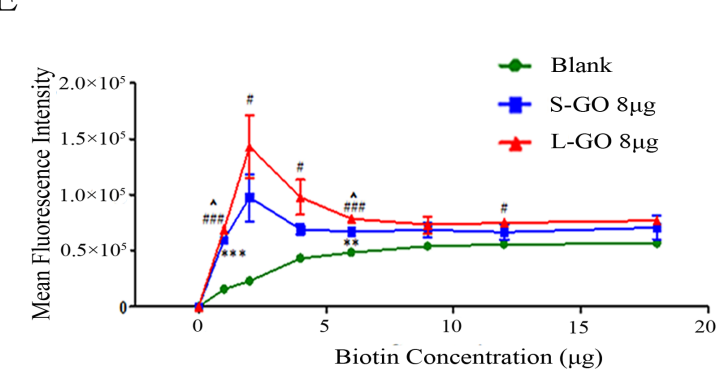

$\mathrm{F}$

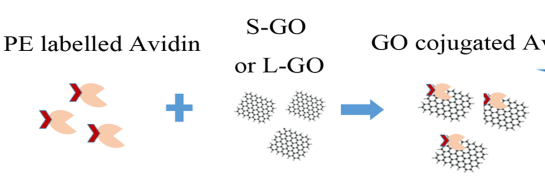

b)
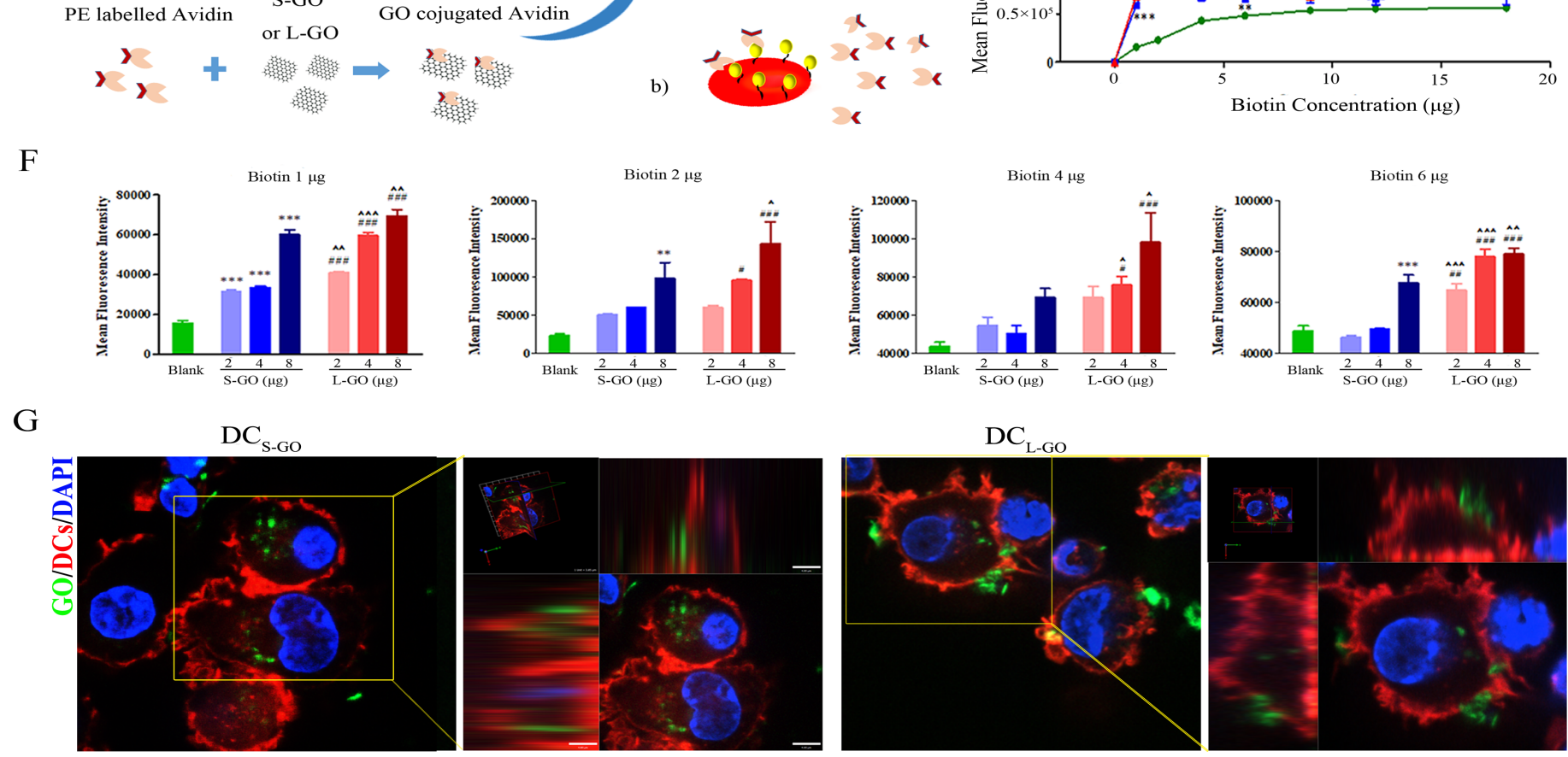

Figure 1

Characterization of graphene oxide and its affinity to cell membrane. A) TEM images of S-GO and L-GO. B) AFM characterization of S-GO and L-GO. C) Confocal Raman mapping of S- or L-GO treated DCs at a dose of $8 \mu \mathrm{g} / \mathrm{mL}$. The DC pinning area is listed on left panel, heatmap of S- or L-GO distribution exhibit in 
middle panel, the Raman spectra of indicated positions are presented in right panel. D)The schematically process of S- or L-GO treated avidin conjugation with biotinized RBCs. E) The specific combination of Sor L-GO $(8 \mu \mathrm{g})$ pretreated PE-avidin with biotinized RBCs of different concentration $(0,1,2,4,6,8,12$ and $16 \mu \mathrm{g})$. F) The specific binding of biotinized RBCs with PE-avidin incubated with S- or L-GO of different concentrations $(2,4$ and $8 \mu \mathrm{g})$. G) Confocal imaging of S- or L-GO location on DCs. Green: FITC-GOs; Red: Rhodamine labeled DCs. ${ }^{*} \mathrm{p}<0.05 \mathrm{~S}-\mathrm{GO}$ compared with Blank. \#p<0.05L-GO compared with Blank. ${ }^{\wedge} \mathrm{p}<$ 0.05 L-GO compared with S-GO.

A

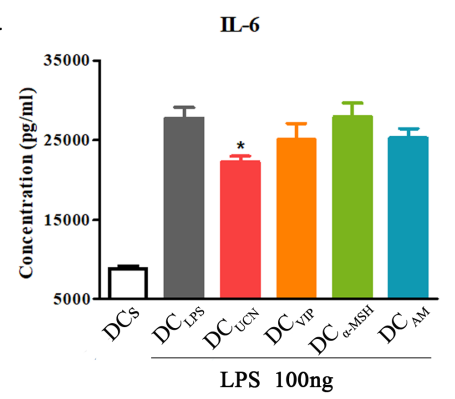

B

T cells:DCs

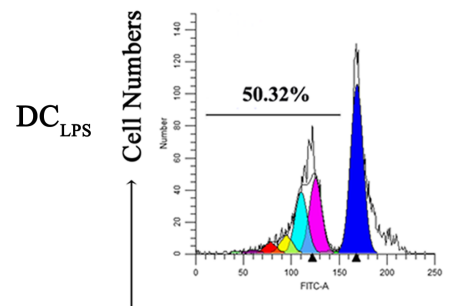

$\mathrm{TDC}_{\mathrm{UCN}}$

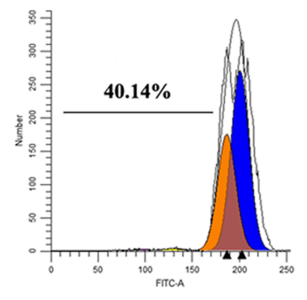

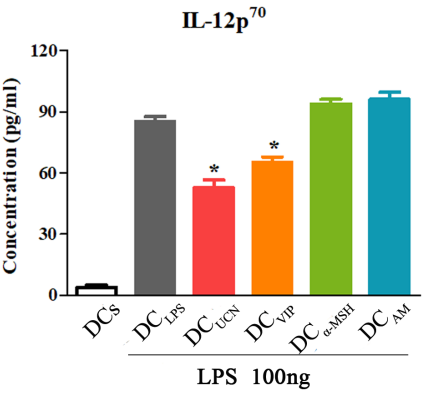
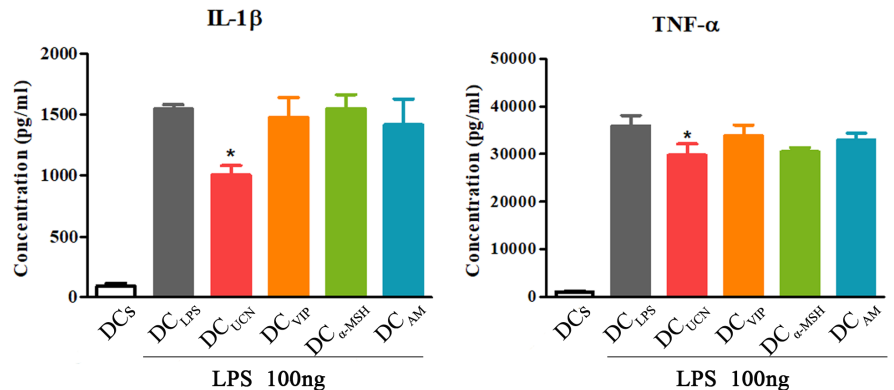

C

$4: 1$

$8: 1$
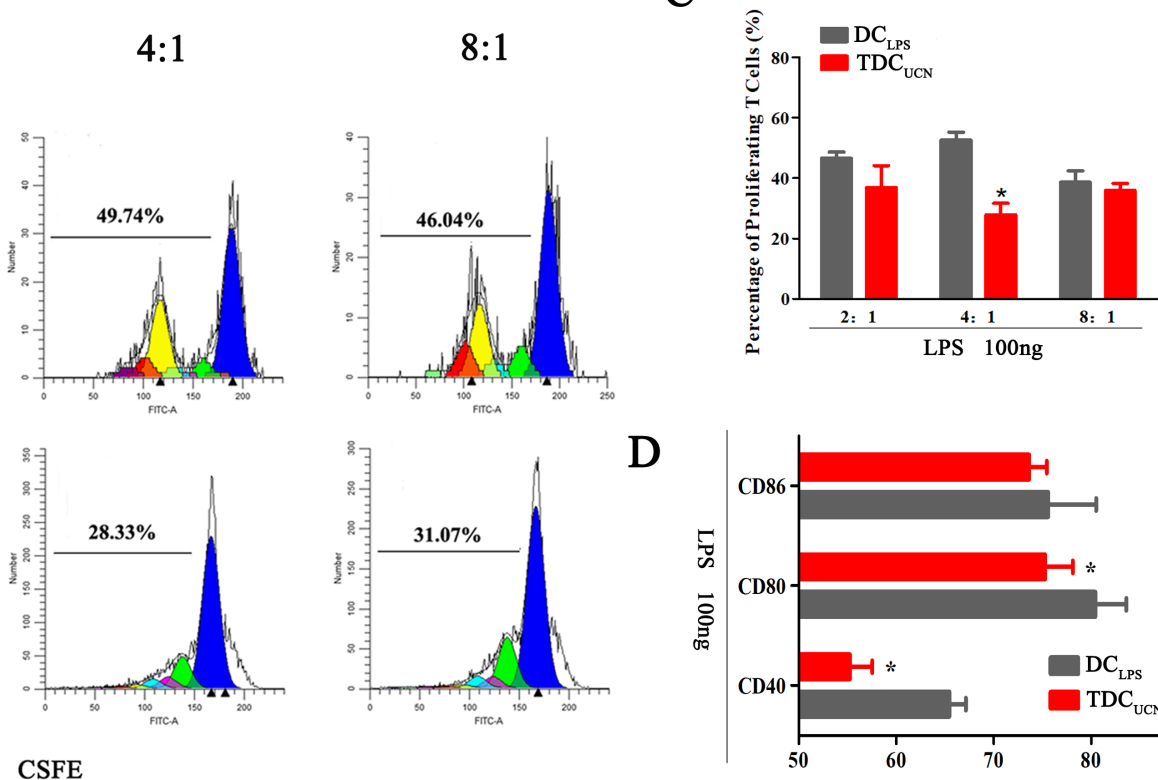

D

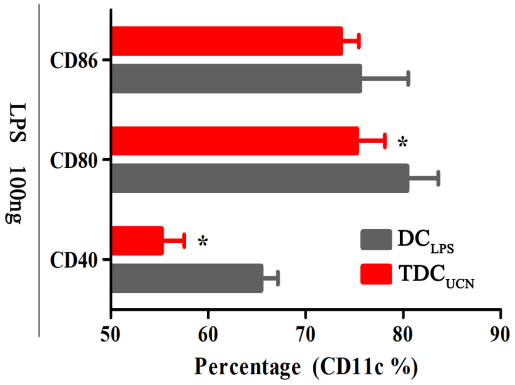

E

F

G
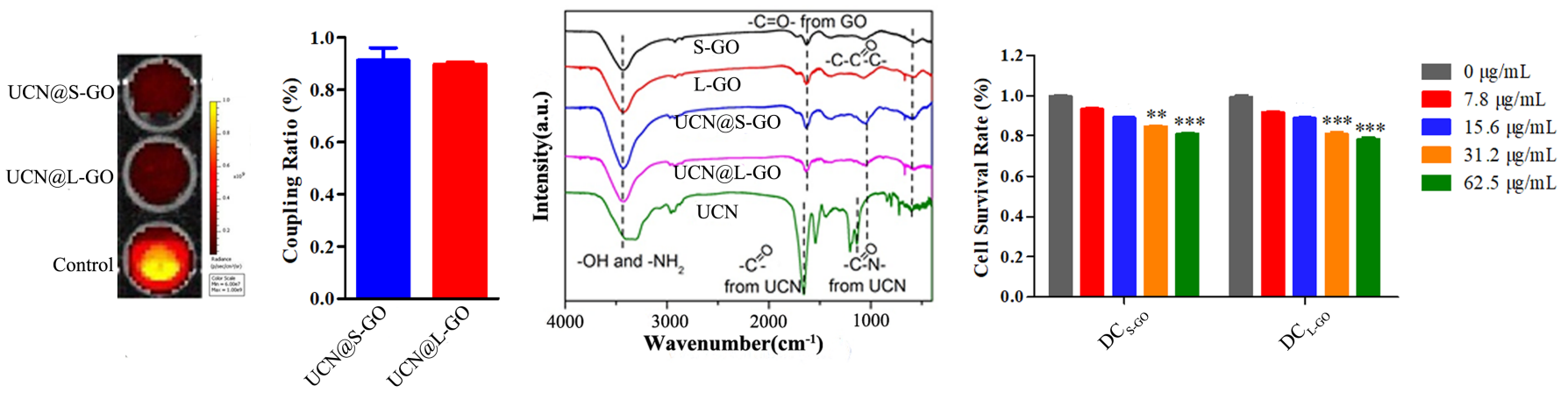

Figure 2 
Design of GO based UCN delivery systems. A) Detection of proinflammatory cytokines IL-12p70, IL-6, IL$1 \beta$ and TNF-a secreted by DCs. ${ }^{*} p<0.05$ compared with DCLPS. B) The proliferation of CD $8+T$ cells is measured after co-culture with neuropeptide pretreated DCs. C) Statistical analysis of CD8+ T cells proliferation. ${ }^{*} p<0.05$ compared with DCLPS. D) Positive percentage of co-stimulatory molecular CD40/80/86 on DCs after neuropeptide incubation. ${ }^{*} p<0.05$ compared with DCLPS. E) The conjugate efficiency of Cy 5 labeled UCN and S- or L-GO by Living imaging system. F) The absorption peak of S- or LGO treated UCN. G) Apoptosis detection of DCs after being co-cultured with S- or L-GO (dose ranged from $0-32 \mu \mathrm{g}$ ) for $48 \mathrm{~h} .{ }^{*} \mathrm{p}<0.05$ compared with $0 \mu \mathrm{g}$ group. 
A

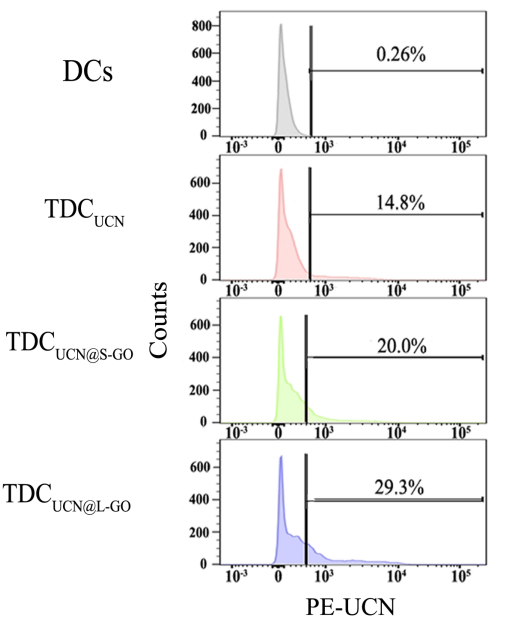

C

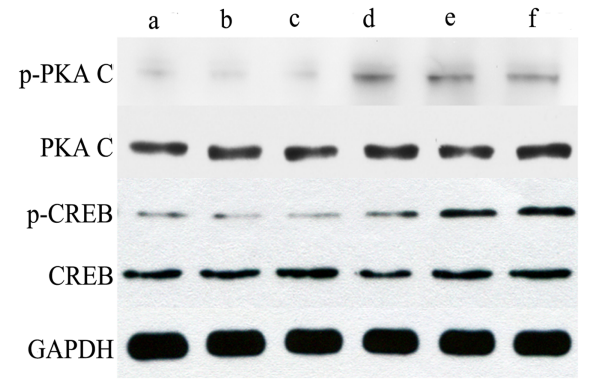

$\mathrm{D}$

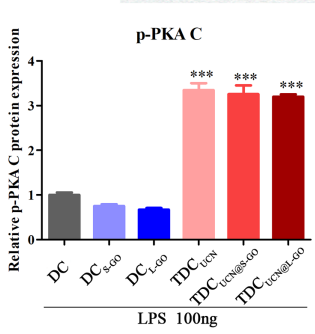

$\mathrm{E}$
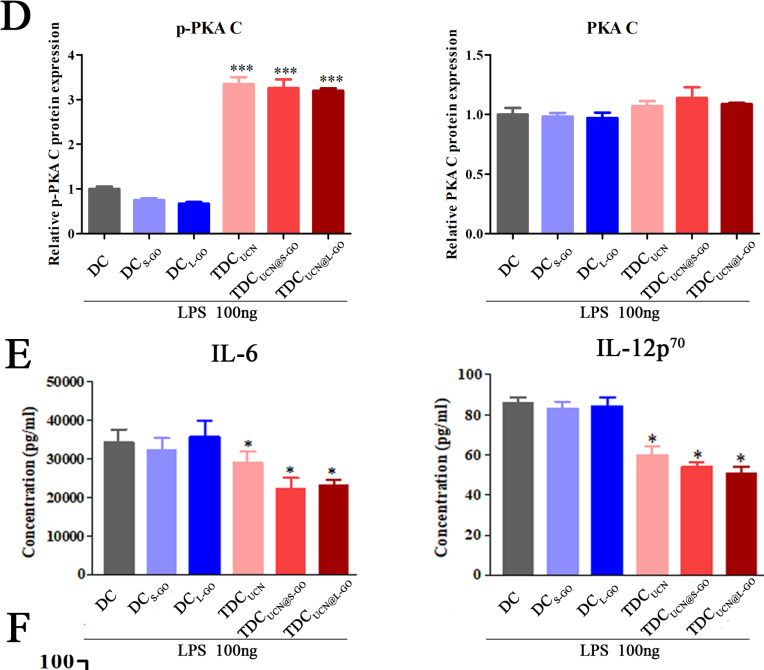

$\mathrm{IL}-12 \mathrm{p}^{70}$
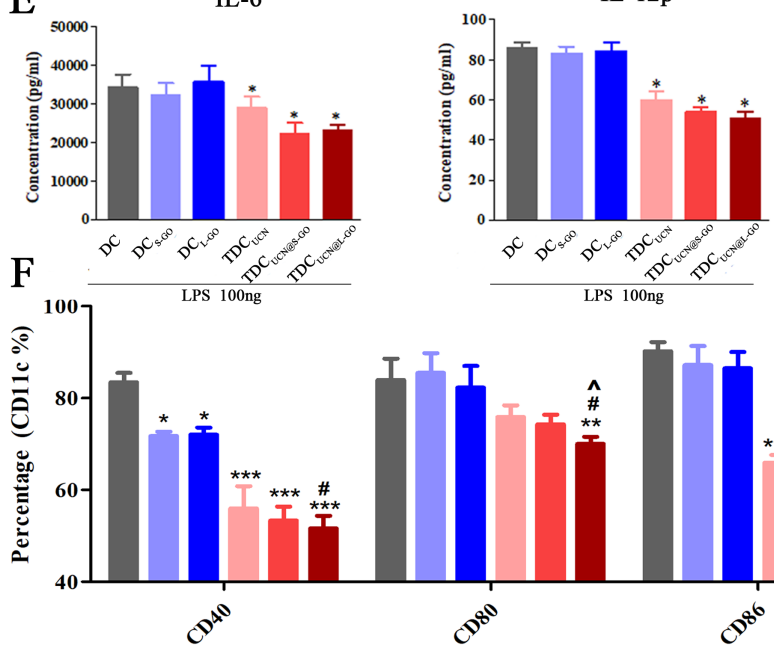

B
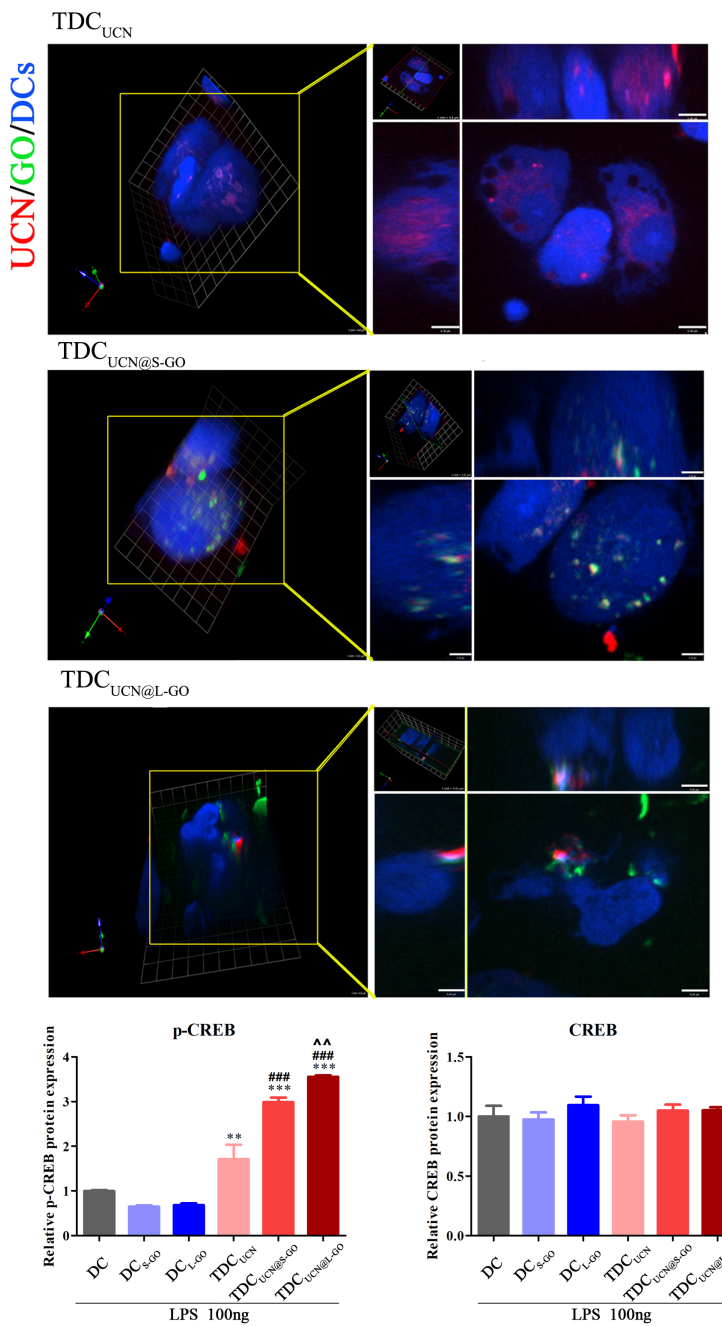

IL-1 $\beta$
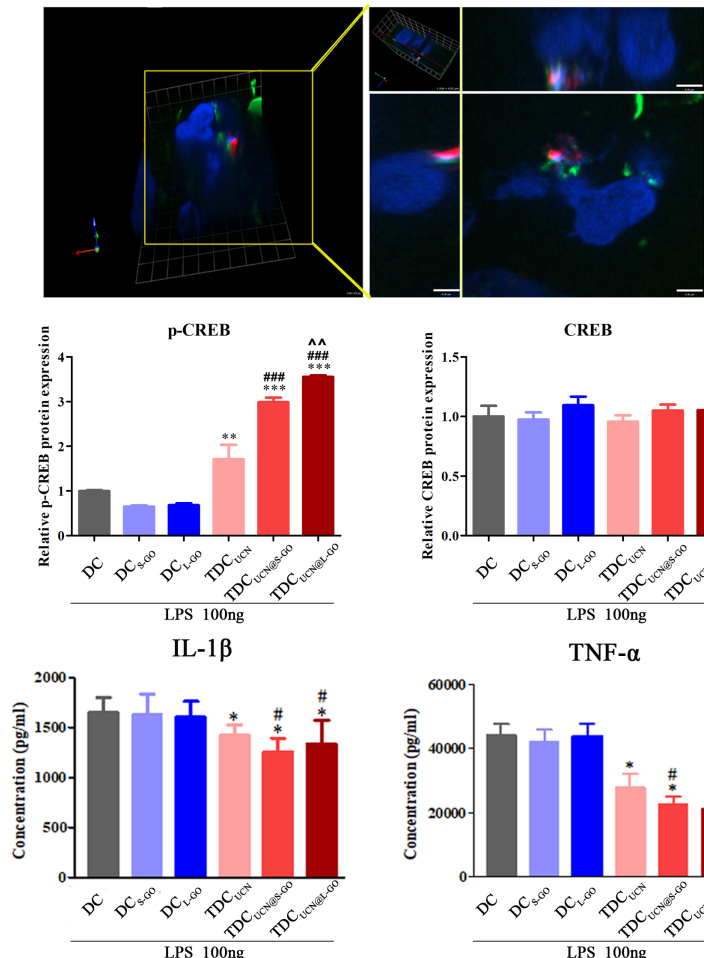

CREB

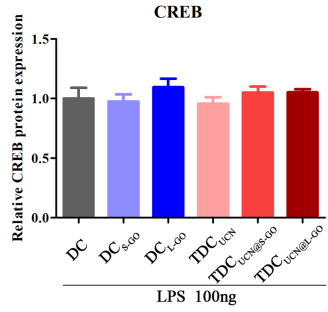

TNF- $\alpha$

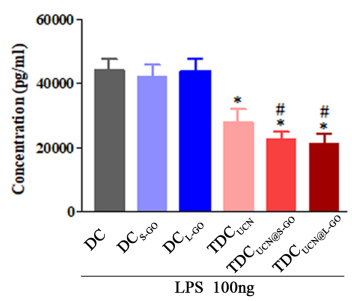

\section{Figure 3}

UCN effectively delivered with large-sized GO to DCs and generated TDCs. A) Detection for fluorescence on DCs pretreated with free 5-TAMRE labeled UCN, UCN@S-GO or UCN@L-GO after 48h. B) Confocal imaging of UCN@GO complex location on DC cytomembrane. Green: FITC labeled S- or L-GO. Red: 5TAMRE labeled UCN. Blue: DCs stained with Cell TrackerTM Blue. Left panel: 3D constructure forcolocation of free UCN, UCN@S-GO and UCN@L-GO with DCs separately. Right panel: partial enlargement. 
C)Western blot analysis for phosphorylation of PKA C/CREB pathway. a: DCLPS; b: DCS-GO; c: DCL-GO; d: TDCUCN; e: TDCUCN@S-GO; f: TDCUCN@L-GO.D) Analysis of the expression level of each protein through grey value. E) Measurements for cytokines secreted by DCs pretreated with GOs, free UCN and UCN@GOs using ELISA.F) Detection for co-stimulatory molecules CD40/80/86 expression on DC cytomembrane. ${ }^{*} p<0.05$ compared with DCs plus 100ng LPS. \#p< 0.05 compared with TDCUCN. ${ }^{\wedge} p<$ 0.05 compared with TDCUCN@S-GO.

A
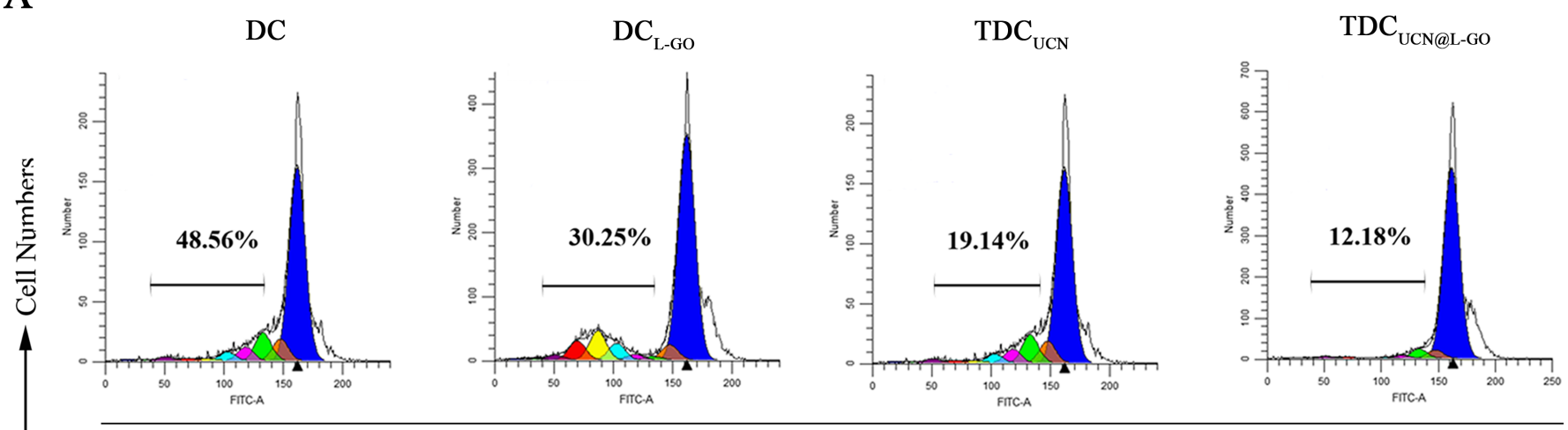

LPS 100ng

CSFE

B

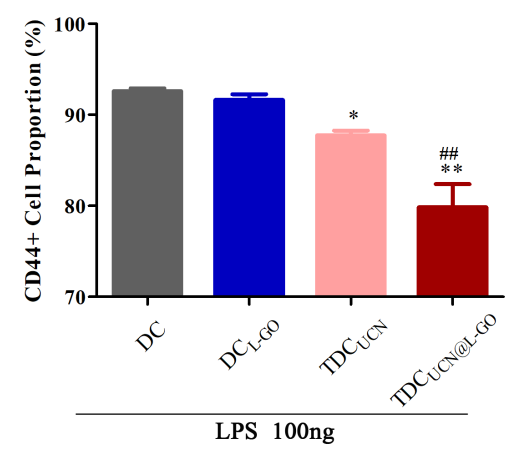

$\mathrm{C}$

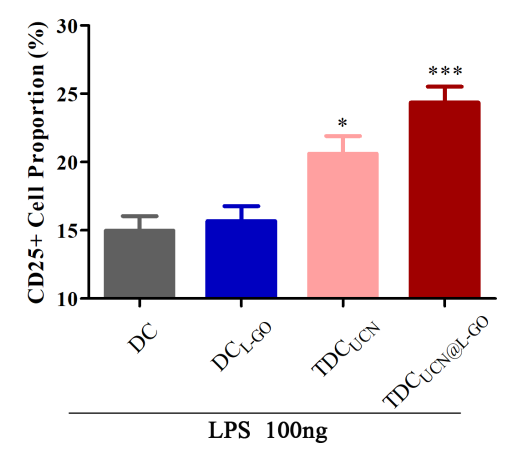

CD8+CD69+

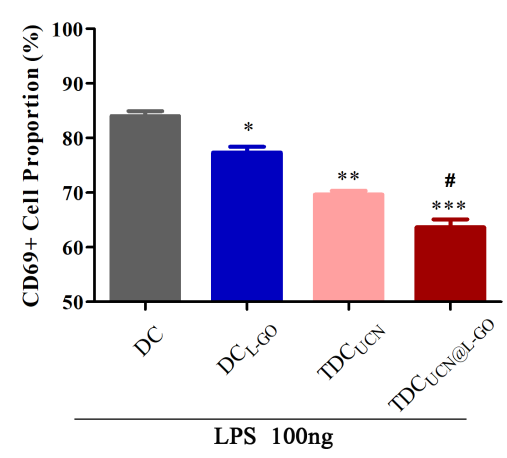

CD4+CTLA4+

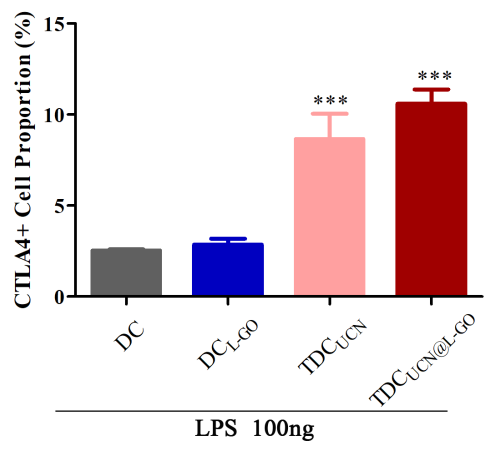

CD8+CD107a+

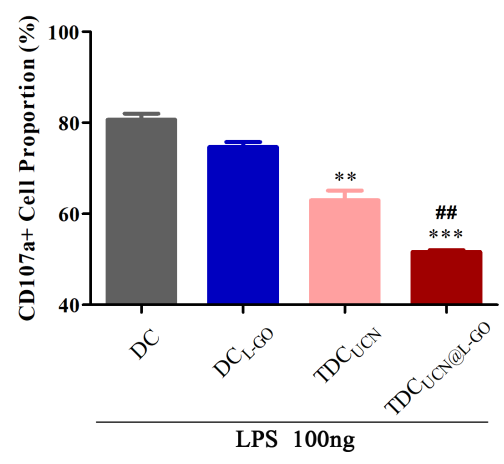

CD4+CD154+

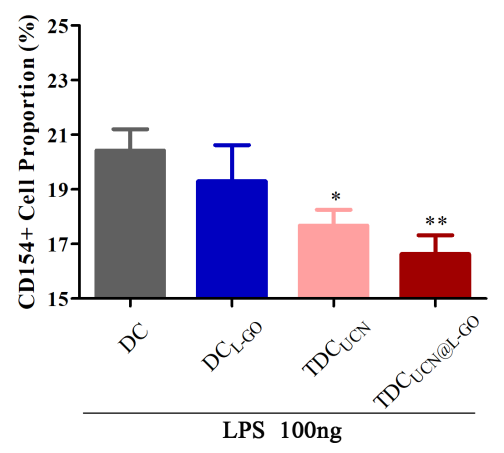

\section{Figure 4}

TDCUCN@L-GO effectively deleted cytotoxic T cells and generated Tregs. A) Proliferation of CD8+ T cells co-cultured with DCs pretreated in various ways for $72 \mathrm{~h}$. B) Analysis of CD8+ T cell activation after incubation with DCs pretreated with GOs, free UCN and UCN@GOs by FACS, including CD25, CD69 and 
CD107a. C) Analysis of CD4+ Treg cell marker after incubation with TDCs pretreated with GOs, free UCN and UCN@GOs by FACS, including CD25, CTLA4 and CD154. * $p<0.05$ compared with DC plus 100ng LPS. \#p< 0.05 compared with TDCUCN.

A

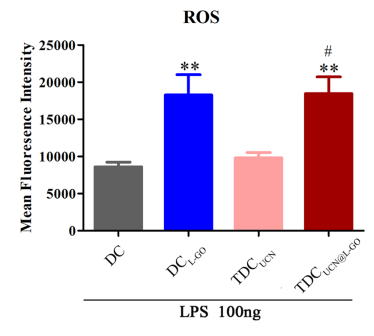

B

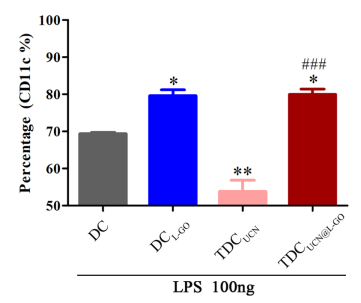

C
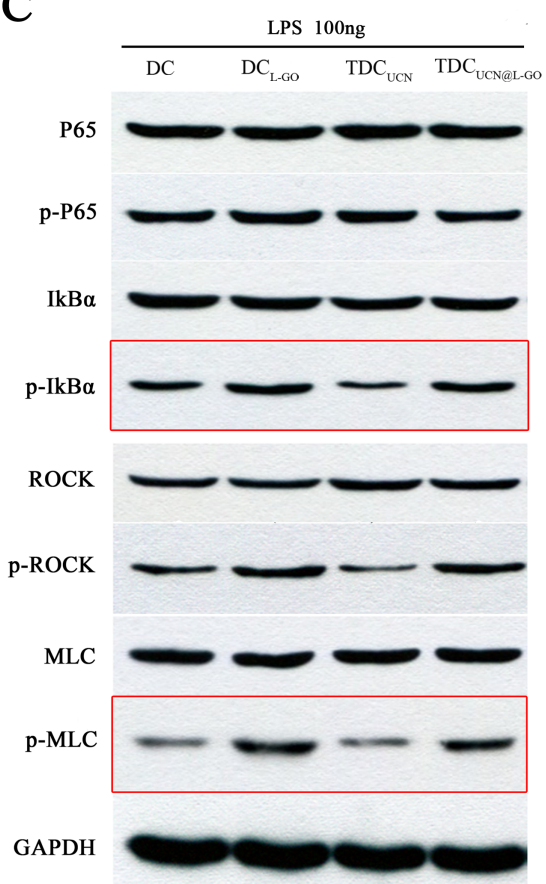

D

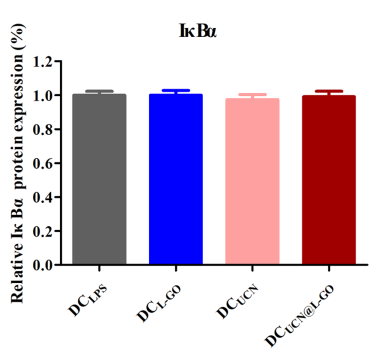

$\mathrm{E}$
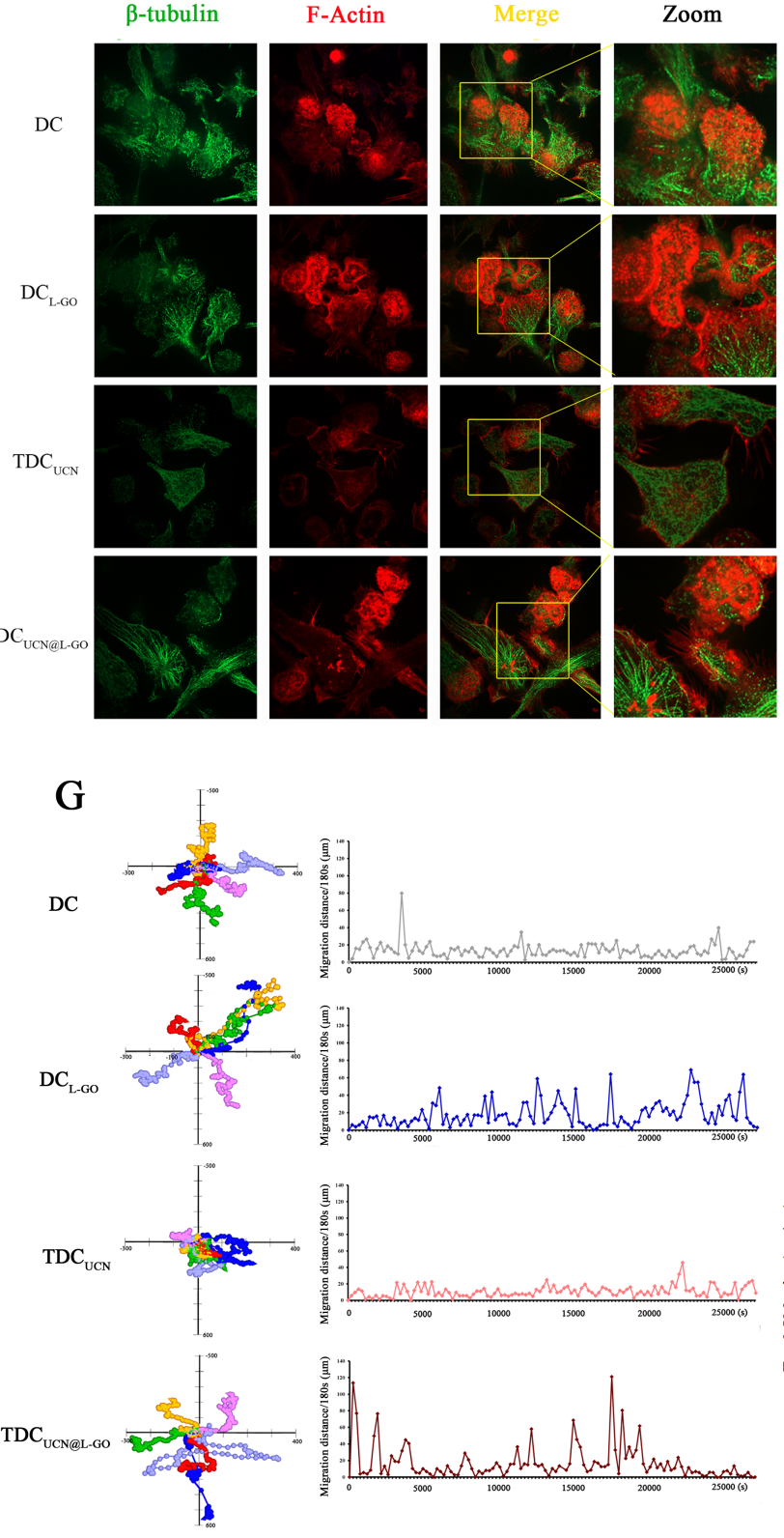

$\mathrm{F}$
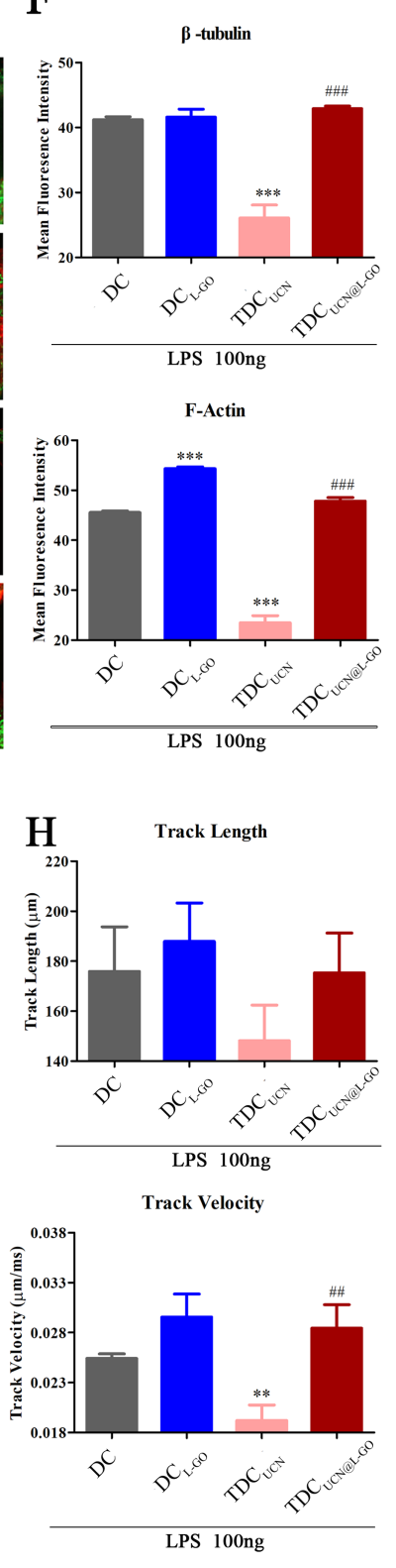

\section{Figure 5}

UCN@L-GO complex accelerated DCs cytoskeleton rearrangement through Rho A/ROCK signal. A) ROS accumulation in DCs after being co-cultured with UCN@L-GO. B) FACS analysis for CCR7 expression on 
DCs. C) Activation detection of NF-KB/IKB and Rho A/ROCK pathway in DCs. The phosphorylation level of IKB and MLC were measured and list in D). E) Confocal imaging of DCs after incubation with L-GO, free UCN and UCN@L-GO for 48h. Green: $\beta$-tubulin. Red: F-Actin. Yellow: merge. The mean fluorescence intensity was measured and listed on F). G) Moving track of DC motility traced by laser confocal. Left panel: moving track. Middle panel: migration distance per 180s. H) Analysis of track length and track velocity were presented by Velocity software. ${ }^{*} p<0.05$ compared with DC plus $100 \mathrm{ng}$ LPS. \#p< 0.05 compared with TDCUCN.

A

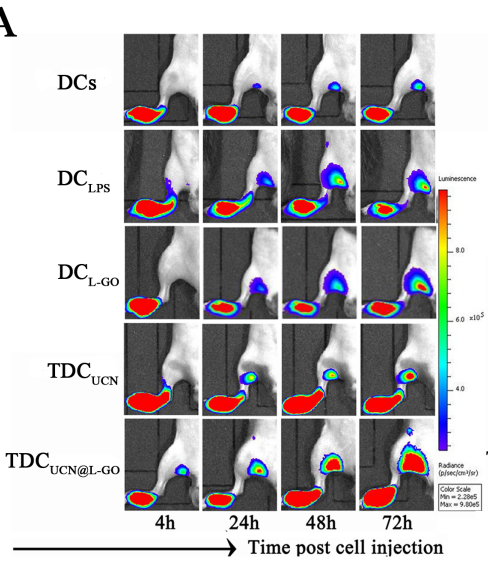

C

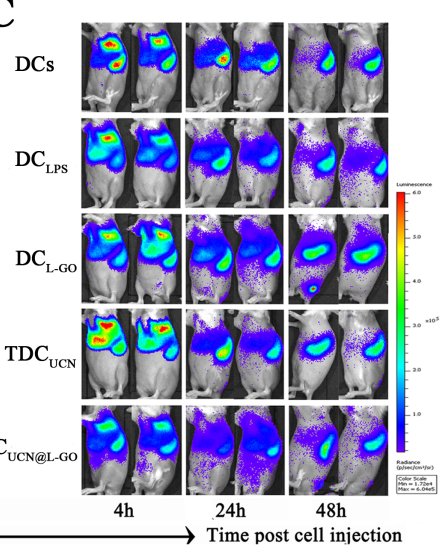

B

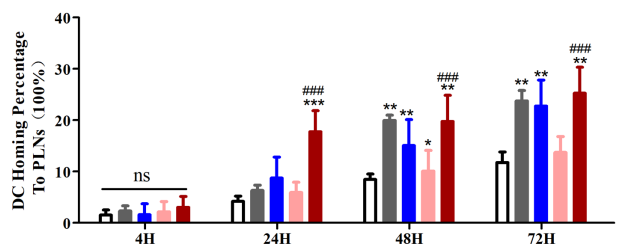

D

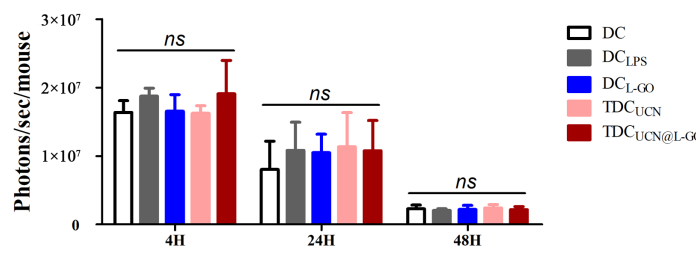

E
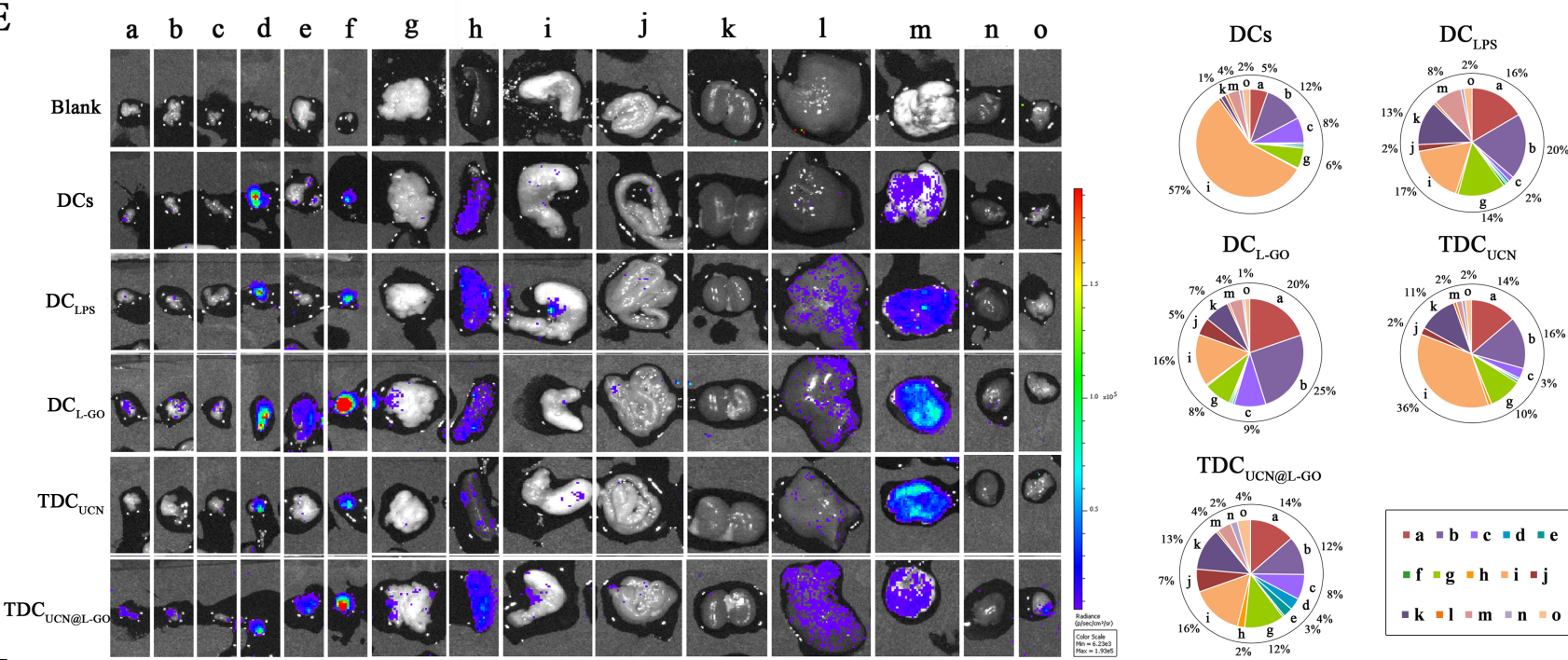

F
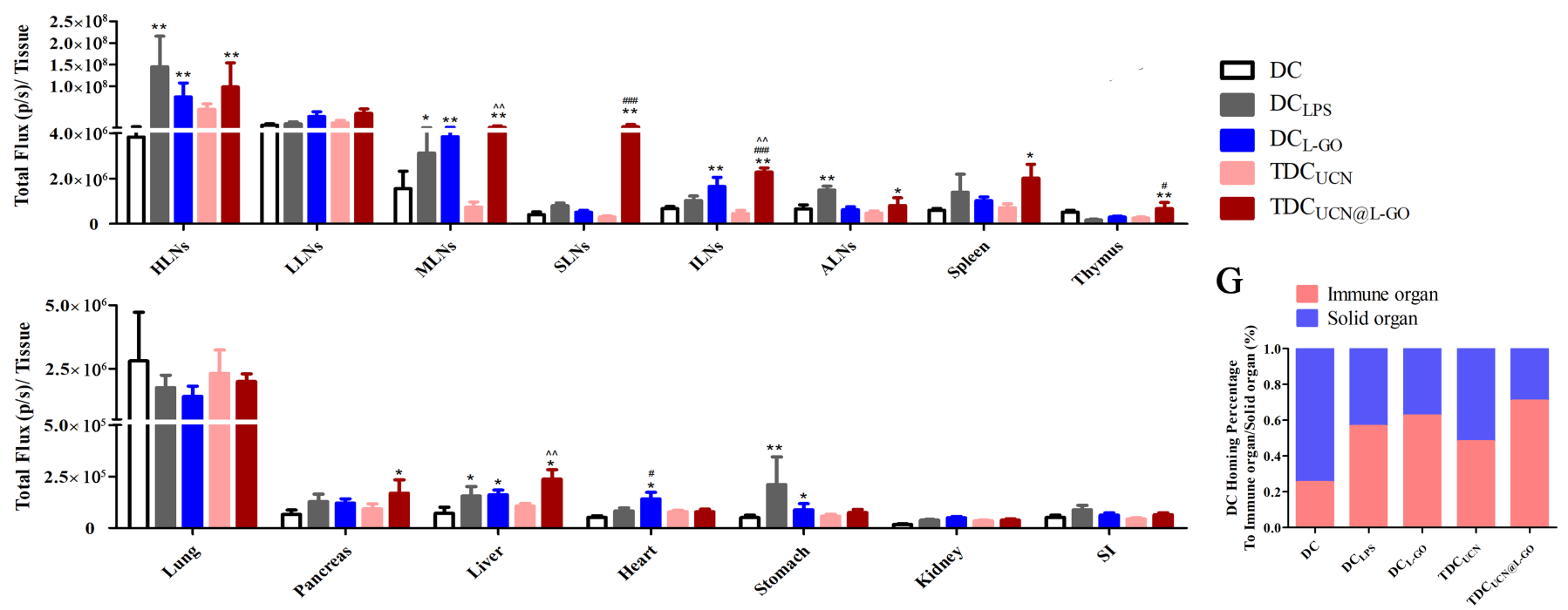


\section{Figure 6}

UCN@L-GO complex promoted DC homing in vivo. A) Evaluation of DCs homing ability to local lymph nodes by footpad injection model. Fluc+ DCs pretreated with L-GO, free UCN or UCN@L-GO was injected subcutaneously and observed at $4 \mathrm{~h}, 24 \mathrm{~h}, 48 \mathrm{~h}$ and $72 \mathrm{~h}$ post injection. B) Analysis of DC homing percentage to PLNs by the fluorescence intensity. C) Distribution of DCs pretreated with various ways after intravenously injection in vivo. Fluorescence signals were detected by Living imaging system at 24h, $48 \mathrm{~h}$ and $72 \mathrm{~h}$ post injection. D) Analysis of DC fluorescence signals at multiple time points. E) Detection of organ fluorescence intensity $72 \mathrm{~h}$ after injection. Left panel: representative image of each organ. Right panel: distribution percentage of DCs for each organ. a: HLNs. b: LLNs. c: MLNs. d: SLNs. e: ILNs. f: ALNs. g: pancreases. h: spleen. i: stomach. j: small intestine. k: kidneys. l: liver. m: lung. n: heart. o: thymus. F) Analysis for fluorescence intensity of each organ. G) Ratio of TDCs homing to immune organs to solid organs. ${ }^{*} p<0.05$ compared with DC plus 100ng LPS. \#p< 0.05 compared with TDCUCN. 
A

C
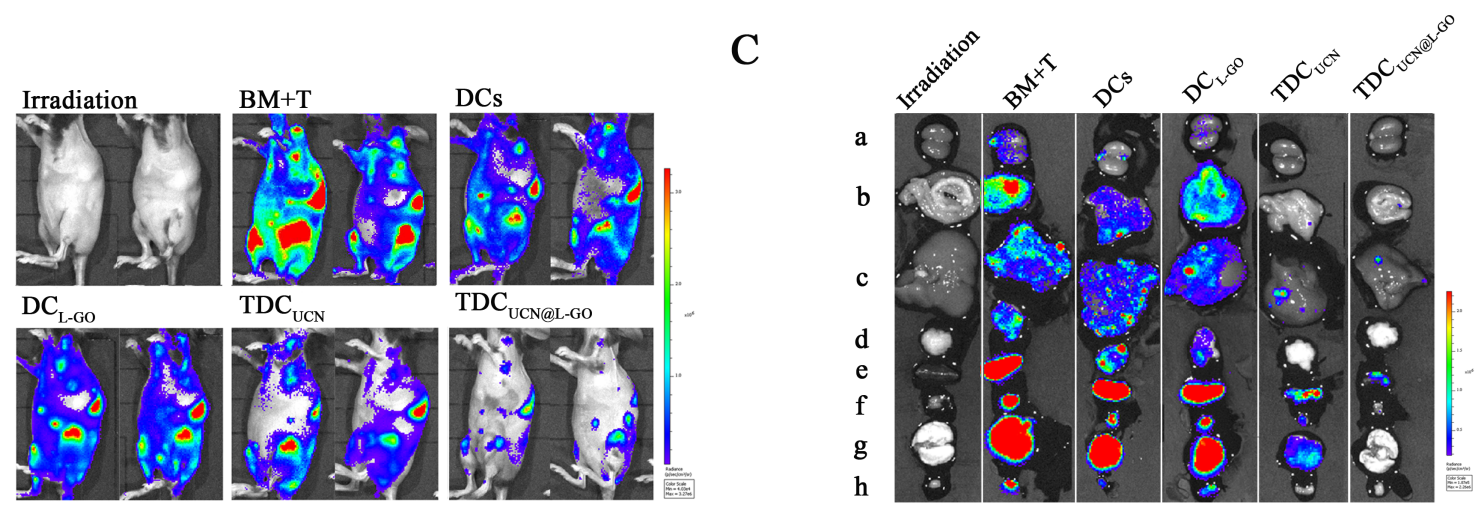

B
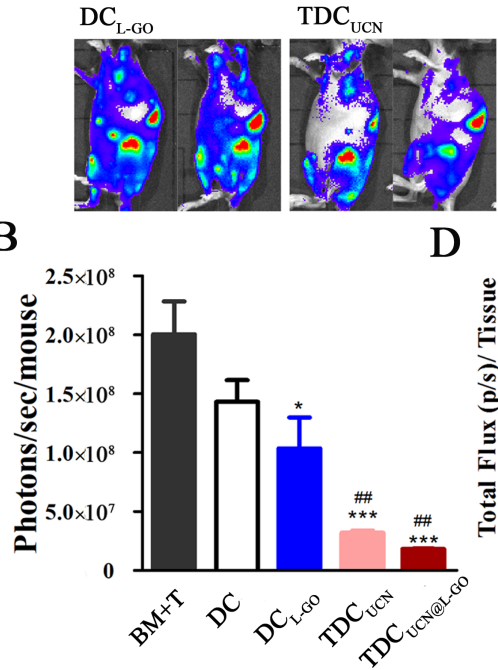

D $\quad 1.5 \times 10^{\circ}$

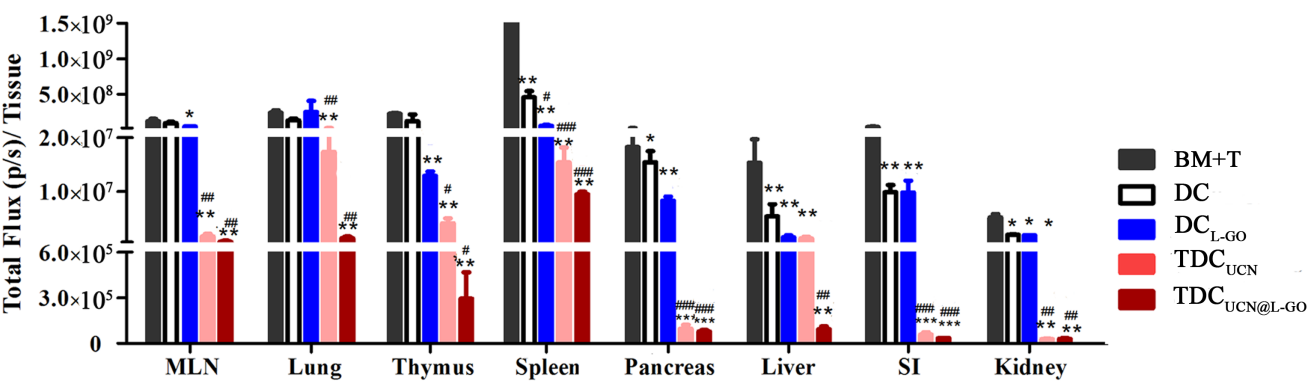

E
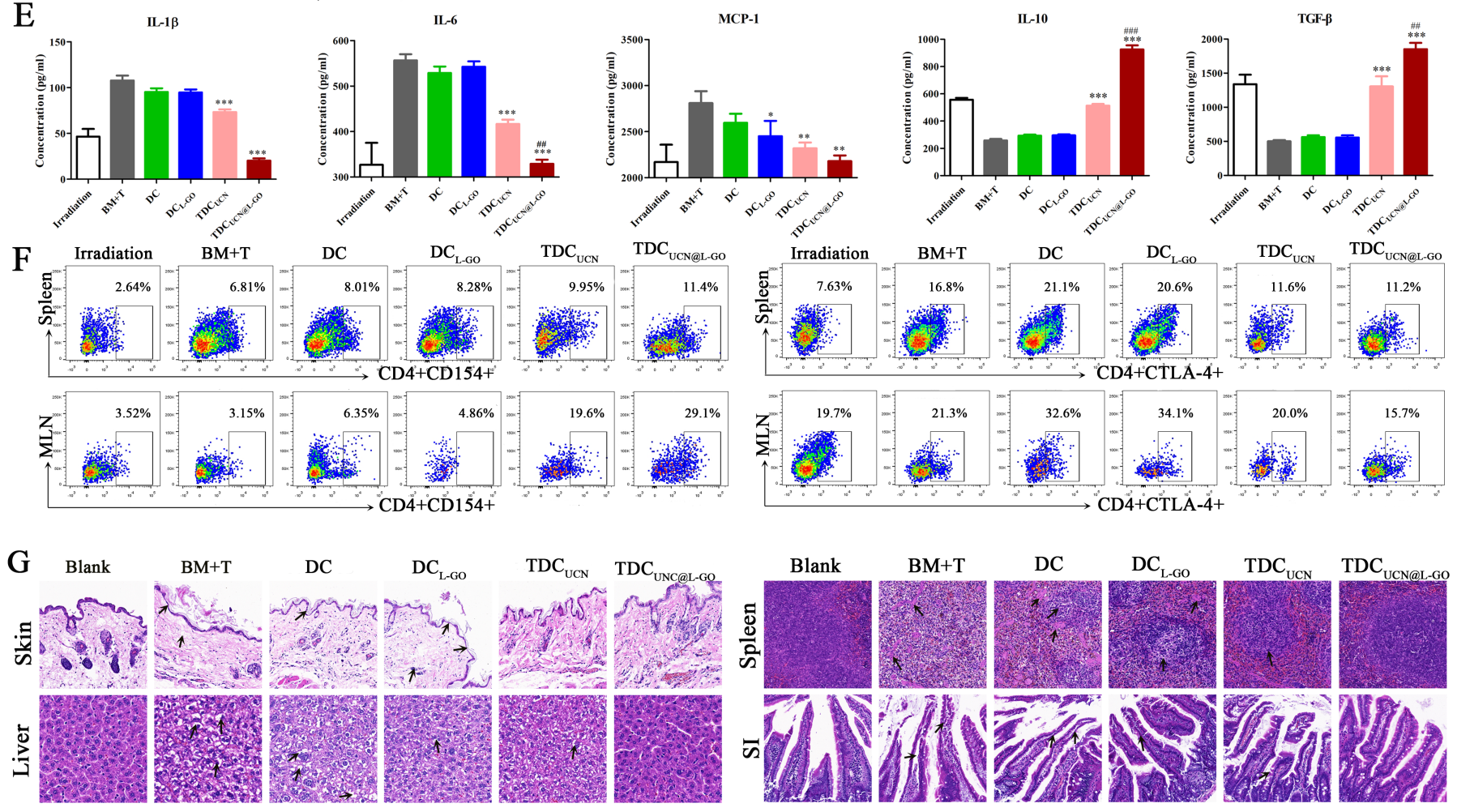

\section{Figure 7}

Strong inhibition of transplanted T cells activation by UCN@L-GO-generated TDCs in acute GVHD. A) The entirety fluorescence intensity was measured by Living imaging system. Fluc+ T cell proliferation was detected by fluorescence signals at 14d. B) Analysis of transplanted T cell activation after injection of DCs pretreated with L-GO, free UCN and UCN@L-GO at 14d. C) Activation of transplanted T cells in each organ after DC-treatment with intravenous injection in vivo. a: kidneys. b: small intestine. c: liver. d: 
thymus. e: spleen. f: pancreases. g: lung. h: MLNs. D) Analysis of fluorescence signals for each organ after injection of DCs pretreated in various ways at 14d.E) Measurements of proinflammatory cytokines in serum by ELISA, including IL-1 $\beta$, IL-6 and MCP-1, and anti-inflammatory ones, including IL-10 and TGFß. F) FAC detection of Treg proportion in spleen and MLNs through CD154 and CTLA-4. G) Pathology analysis of organs by HE staining, including spleen, liver, skin and small intestine. Arrow indicated the damage position caused by cytotoxic T cell infiltration. ${ }^{*} \mathrm{p}<0.05$ compared with DC plus $100 \mathrm{ng}$ LPS. \#p< 0.05 compared with TDCUCN.

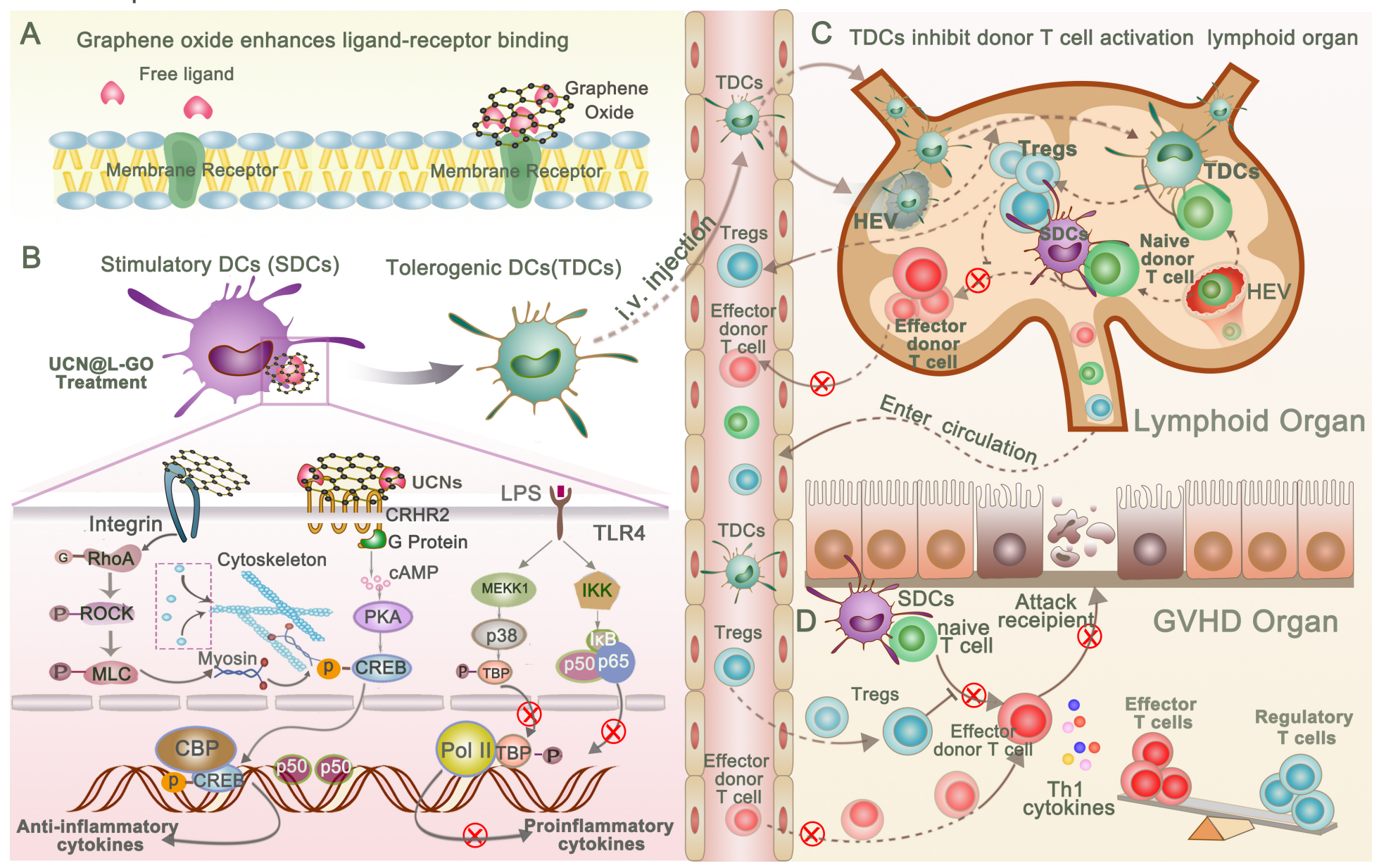

\section{Figure 8}

Schematic of the overall process. A) Graphene oxide enhances receptor-ligand combination; B) Pathway phosphorylation triggered by UCN@L-GO; C) TDCs homing to lymopiod organ through high endothelial venule (HEV) to inhibit effector donor T cells activation; D) Modulation of T cell phenotype in GVHD targeted organs.

\section{Supplementary Files}

This is a list of supplementary files associated with this preprint. Click to download.

- SupplementaryInformation.docx 